\title{
Quakebend deformations in complex hyperbolic quasi-Fuchsian space
}

\author{
IOANNIS D PLATIS
}

\begin{abstract}
We study quakebend deformations in complex hyperbolic quasi-Fuchsian space $\mathcal{Q}_{\mathbb{C}}(\Sigma)$ of a closed surface $\Sigma$ of genus $g>1$, that is the space of discrete, faithful, totally loxodromic and geometrically finite representations of the fundamental group of $\Sigma$ into the group of isometries of complex hyperbolic space. Emanating from an $\mathbb{R}$-Fuchsian point $\rho \in \mathcal{Q}_{\mathbb{C}}(\Sigma)$, we construct curves associated to complex hyperbolic quakebending of $\rho$ and we prove that we may always find an open neighborhood $U(\rho)$ of $\rho$ in $\mathcal{Q}_{\mathbb{C}}(\Sigma)$ containing pieces of such curves. Moreover, we present generalisations of the well known Wolpert-Kerckhoff formulae for the derivatives of geodesic length function in Teichmüller space.
\end{abstract}

$32 \mathrm{G} 05 ; 32 \mathrm{M} 05$

\section{Introduction and statement of results}

There are several structures which can be assigned to a closed surface $\Sigma$ of genus $g>1$. Let us denote by $X$ one of the following spaces: a) the hyperbolic plane $\mathbf{H}_{\mathbb{R}}^{2}$, b) the hyperbolic space $\mathbf{H}_{\mathbb{R}}^{3}$ and c) the complex hyperbolic plane $\mathbf{H}_{\mathbb{C}}^{2}$. Also by $G$ we denote the group of isometries $\operatorname{Isom}(X)$. Suppose that $\rho_{0}$ is a discrete, faithful, injective homomorphism of the fundamental group $\pi_{1}=\pi_{1}(\Sigma)$ into $G$.

(a) If $G=\operatorname{Isom}\left(\mathbf{H}_{\mathbb{R}}^{2}\right)=\operatorname{PSL}(2, \mathbb{R})$ then $\rho_{0}$ defines a hyperbolic structure on $\Sigma$ : the group $\Gamma_{0}=\rho_{0}\left(\pi_{1}\right)$ is Fuchsian and the set $\Sigma_{0}=\mathbf{H}_{\mathbb{R}}^{2} / \Gamma_{0}$ is a hyperbolic surface. The set of all hyperbolic structures of $\Sigma$ is the Teichmüller space $\mathcal{T}(\Sigma)$ of $\Sigma$. Since a hyperbolic structure on a surface yields a conformal structure and vice versa, $\mathcal{T}(\Sigma)$ may be also thought as the set of all conformal structures of $\Sigma$.

(b) If $G=\operatorname{Isom}\left(\mathbf{H}_{\mathbb{R}}^{3}\right)=\operatorname{PSL}(2, \mathbb{C})$ then $\rho_{0}$ defines a quasi-Fuchsian structure on $\Sigma$ : the group $\Gamma_{0}=\rho_{0}\left(\pi_{1}\right)$ is quasi-Fuchsian and the set $M_{0}=\mathbf{H}_{\mathbb{R}}^{3} / \Gamma_{0}$ is a quasi-Fuchsian manifold, that is a hyperbolic 3-manifold isomorphic to $\Sigma \times(0,1)$. The set of all quasi-Fuchsian structures of $\Sigma$ is the real hyperbolic quasi-Fuchsian space $\mathcal{Q}_{\mathbb{R}}(\Sigma)$ of $\Sigma$. This is the complexification of $\mathcal{T}(\Sigma)$ and thus points of $\mathcal{T}(\Sigma)$ may be viewed as the diagonal of $\mathcal{Q}_{\mathbb{R}}(\Sigma)$. 
(c) If $G=\operatorname{Isom}\left(\mathbf{H}_{\mathbb{C}}^{2}\right)=\mathrm{PU}(2,1)$ then $\rho_{0}$ defines a complex hyperbolic quasiFuchsian structure on $\Sigma$ : the group $\Gamma_{0}=\rho_{0}\left(\pi_{1}\right)$ is complex hyperbolic quasiFuchsian and the set $M_{0}=\mathbf{H}_{\mathbb{C}}^{2} / \Gamma_{0}$ is a complex hyperbolic quasi-Fuchsian manifold $M_{0}$, that is a complex hyperbolic 4-manifold isomorphic to a disc bundle over $\Sigma$ (Goldman-Kapovich-Leeb [5]). The set of all complex hyperbolic quasi-Fuchsian structures of $\Sigma$ is the complex hyperbolic quasi-Fuchsian space $\mathcal{Q}_{\mathbb{C}}(\Sigma)$ of $\Sigma$.

A deformation of $\rho_{0}$ is a curve $\rho_{t}=\rho(t)$ such that $\rho(0)=\rho_{0}$. Deformations in Teichmüller and real quasi-Fuchsian spaces are very well known and have been studied extensively, at least throughout the last thirty years. This is in contrast to complex hyperbolic case where up to the present, very little is known. In this article we try to shed some light on the complex hyperbolic case, by studying a basic deformation in complex hyperbolic quasi-Fuchsian space $\mathcal{Q}_{\mathbb{C}}(\Sigma)$. Since our motivation comes from the classical cases a) and b), we wish to review in brief some basic facts about them. In the case of Teichmüller space $\mathcal{T}(\Sigma)$, the basic deformation is the Fenchel-Nielsen $(\mathrm{F}-\mathrm{N})$ deformation; a thorough study of this has been carried out by Wolpert in [18]. We cut $\Sigma_{0}$ along a simple closed geodesic $\alpha$, rotate one side of the cut relative to the other and attach the sides in their new position. The hyperbolic metric in the complement of the cut extends to a hyperbolic metric in the new surface. In this way a deformation $\rho_{t}$ (depending on the free homotopy class of $\alpha$ ) is defined and its infinitesimal generator $t_{\alpha}$ is the F-N vector field. Such vector fields are very important: at each point of $\mathcal{T}(\Sigma), 6 g-6$ of such fields form a basis of the tangent space. Moreover, the WeilPetersson Kähler form of $\mathcal{T}(\Sigma)$ may be described completely in terms of the variations of geodesic length of simple closed geodesics under the action of these fields. The basic formula for this is Wolpert's first derivative formula: If $\alpha, \beta$ are simple closed geodesics in $\Sigma_{0}, l_{\alpha}$ is the geodesic length of $\alpha$ and $t_{\beta}$ is the $\mathrm{F}-\mathrm{N}$ vector field associated to $\beta$ then at the point $\rho_{0}$ we have

$$
t_{\beta} l_{\alpha}=\sum_{p \in \alpha \cap \beta} \cos \left(\phi_{p}\right),
$$

where $\phi_{p}$ is the oriented angle of intersection between $\alpha$ and $\beta$ at $p$. Another basic formula concerns the mixed variations $t_{\beta} t_{\gamma} l_{\alpha}$; the reader should see for instance Wolpert [19] or [18] for details.

The concept of bending was inspired by the following question: what happens if instead of cut and rotate the surface along a closed geodesic and then glue the pieces back again, we "bend" the surface along this geodesic in an angle $\phi$ ? This question was primarily examined in Thurston's Mickey Mouse example in [15]: given a hyperbolic structure on a closed surface of genus 2 , we consider the structure arising from the 
bending of the surface along a simple closed geodesic by an angle $\pi / 2$. If the geodesic is small enough, then the resulting deformation gives rise to a structure on the surface which is no longer hyperbolic but rather quasi-Fuchsian; it realises a quasi-Fuchsian representation of $\pi_{1}$ into $\operatorname{PSL}(2, \mathbb{C})$, that is an element of $\mathcal{Q}_{\mathbb{R}}(\Sigma)$. Thurston's idea was extended later for closed surfaces of arbitrary genus $g>1$ by Kourouniotis and Epstein-Marden.

In [7] Kourouniotis, working in the spirit of Wolpert's construction of the F-N deformation, constructs a quasiconformal homeomorphism of the complex plane which he calls the bending homeomorphism. Given a hyperbolic structure on $\Sigma$, from this homeomorphism he obtains a quasi-Fuchsian structure on $\Sigma$. We note that the bending homeomorphism is then extended naturally to higher dimensions to produce discrete representations of the fundamental group of a closed surface into the group of isometries of $n$-dimensional hyperbolic space $\mathbf{H}_{\mathbb{R}}^{n}$.

Epstein and Marden took a different and much more general point of view in [2]. Given a hyperbolic structure $\rho_{0}$ on a closed surface $\Sigma$, then for every discrete geodesic lamination $\Lambda$ in $\Sigma$ with complex transverse measure $\mu$ and a simple closed geodesic $\alpha$ in $\Sigma_{0}$, there exists an isometric map $h$, depending on $\alpha$, of $\Sigma_{0}$ into a hyperbolic 3-manifold $M_{h}$ (the quakebend map). The image of this map is a pleated surface $\Sigma_{h}$, that is a complete hyperbolic surface which may be viewed as the original surface bent along the leaves of the lamination in angles depending on the imaginary part of $\mu$, with its flat pieces translated relative to the leaves in distances depending on the real part of $\mu$. The pleated surface $\Sigma_{h}$ is then the boundary of the convex hull of $M_{h}$. For small $t \in \mathbb{C}$, quakebending along $\Lambda$ with transverse measure $t \mu$ produces injective homomorphisms of $\pi_{1}$ into $\operatorname{PSL}(2, \mathbb{C})$ with quasi-Fuchsian image and in this way we obtain a deformation $\rho_{t \mu}$ (the quakebend curve) of quasi-Fuchsian space $\mathcal{Q}_{\mathbb{R}}(\Sigma)$ with initial point our given hyperbolic structure, that is a point in the Teichmüller space $\mathcal{T}(\Sigma)$ of $\Sigma$. It is evident that $\mathrm{F}-\mathrm{N}$ as well as Kourouniotis' bending deformation are special cases of the above construction; the first is induced from the case where $\mu$ is real (pure earthquake) and the second from the case where $\mu$ is imaginary (pure bending). Infinitesimal generators of quakebend curves are the holomorphic vector fields $T_{\mu}$. If $\alpha$ is a simple closed geodesic in $\Sigma_{0}$ and in the case where $\lambda$ is finite with leaves $\gamma_{1}, \ldots, \gamma_{n}$, then at the point $\rho_{0}$ we have

$$
T_{\mu} l_{\alpha}=\frac{d l\left(\rho_{t \mu}\right)}{d t}(0)=\sum_{k=1}^{n} \Re\left(\zeta_{k}\right) \cdot \cos \left(\phi_{k}\right)
$$

where $\zeta_{k}=\mu\left(\alpha \cap \gamma_{k}\right)$ and $\phi_{k}$ are the oriented angles of intersection of $\alpha$ and $\gamma_{k}$. 
This formula is a generalisation of a Kerckhoff's formula when $\mu \in \mathbb{R}$, see [6]. Epstein and Marden also give formulae for the second derivative as well as generalisation of these in the case where $\Lambda$ is infinite.

In [8] Kourouniotis revisits the idea of bending. Based in Epstein-Marden [2] and using his bending homeomorphism as in [7], he constructs quakebending curves in $\mathcal{Q}_{\mathbb{R}}(\Sigma)$ but there, the initial point $\rho_{0}$ is a quasi-Fuchsian structure on $\Sigma$. Moreover in [9] he goes on to define the variations of the complex length $\lambda_{\alpha}$ of a simple closed curve under bending along $(\Lambda, \mu)$. Namely, at $\rho_{0}$ his formula for the first derivative may be written as

$$
T_{\mu} \lambda_{\alpha}=\frac{d l\left(\rho_{t \mu}\right)}{d t}(0)=\sum_{k=1}^{n} \zeta_{k} \cdot \cosh \left(\sigma_{k}\right) .
$$

Again, $\zeta_{k}=\mu\left(\alpha \cap \gamma_{k}\right)$ and $\sigma_{k}$ is the complex distance of $\alpha$ and $\gamma_{k}$. We note that Kourouniotis also presents a formula for the second derivative. Kourouniotis' results enabled the author to describe completely the complex symplectic form of $\mathcal{Q}_{\mathbb{R}}(\Sigma)$ in [13]. This form may be thought as the complexification of the Weil-Petersson symplectic form of $\mathcal{T}(\Sigma)$. We remark finally that generalisations of the derivative formulae were given for instance by Series in [14] and also by Parker and Series in [12].

We now concentrate to the complex hyperbolic setting. Here there are several obstructions. First, and in contrast to the cases of $\mathcal{T}(\Sigma)$ and $\mathcal{Q}_{\mathbb{R}}(\Sigma)$ it is not known whether $\mathcal{Q}_{\mathbb{C}}(\Sigma)$ is open in the representation variety $\mathcal{V}_{\mathbb{C}}(\Sigma)=\operatorname{Hom}\left(\pi_{1}, \operatorname{SU}(2,1)\right) / \operatorname{SU}(2,1)$. This fact is crucial for the construction of deformations lying inside $\mathcal{T}(\Sigma)$ or $\mathcal{Q}_{\mathbb{R}}(\Sigma)$. There is an important invariant of a representation $\rho: \pi_{1} \longrightarrow \mathrm{SU}(2,1)$ called the Toledo invariant denoted $\tau(\rho)$ (see Toledo [16]). (For more information about the Toledo invariant, the reader may consult for instance Parker-Platis [10] and the references given there.) The representation variety $\mathcal{V}_{\mathbb{C}}(\Sigma)$ has $2 \cdot(2 g-2)+1$ components distinguished by this invariant. It is not yet clear how $\mathcal{Q}_{\mathbb{C}}(\Sigma)$ sits inside the representation variety. There are some things known though for Fuchsian representations. There are two ways to make a Fuchsian representation act on $\mathbf{H}_{\mathbb{C}}^{2}$. These correspond to the two types of totally geodesic, isometric embeddings of the hyperbolic plane into $\mathbf{H}_{\mathbb{C}}^{2}$. Namely, totally real Lagrangian planes, which may be thought of as copies of $\mathbf{H}_{\mathbb{R}}^{2}$, and complex lines, which may be thought of as copies of $\mathbf{H}_{\mathbb{C}}^{1}$. If a discrete, faithful representation $\rho$ is conjugate to a representation $\rho: \pi_{1} \longrightarrow \mathrm{SO}(2,1)<\mathrm{SU}(2,1)$ then it preserves a Lagrangian plane and is called $\mathbb{R}-$ Fuchsian. If a discrete, faithful representation $\rho$ is conjugate to a representation $\rho: \pi_{1} \longrightarrow \mathrm{S}(\mathrm{U}(1) \times \mathrm{U}(1,1))<\mathrm{SU}(2,1)$ then it preserves a complex line and is called $\mathbb{C}-F$ uchsian. $\mathbb{C}$-Fuchsian representations are 
the elements of the components $\tau= \pm(2 g-2)$ of $\mathcal{V}_{\mathbb{C}}(\Sigma)$. On the other hand, all $\mathbb{R}$-Fuchsian representations lie inside the component $\tau=0$ of $\mathcal{V}_{\mathbb{C}}(\Sigma)$. It is reasonable to ask whether starting from an $\mathbb{R}$-Fuchsian point, one can find an open neighborhood of this point inside $\mathcal{Q}_{\mathbb{C}}(\Sigma)$. The following Theorem has been proved in [10, Theorem $1.1]$.

Theorem A Let $\Sigma$ be a closed surface of genus $g>1$ with fundamental group $\pi_{1}=$ $\pi_{1}(\Sigma)$. Let $\rho_{0}: \pi_{1} \rightarrow \mathrm{SU}(2,1)$ be an $\mathbb{R}-$ Fuchsian representation of $\pi_{1}$. Then there exists an open neighbourhood $U=U\left(\rho_{0}\right)$ of $\rho_{0}$ in $\operatorname{Hom}\left(\pi_{1} \rightarrow \operatorname{SU}(2,1)\right) / \operatorname{SU}(2,1)$ so that any representation $\rho$ in $U$ is complex hyperbolic quasi-Fuchsian.

This Theorem will enable us to prove our first main result, see below.

In [1], B Apanasov took the point view of Kourouniotis in [7] to construct bending curves in $\mathcal{Q}_{\mathbb{C}}(\Sigma)$. Starting from an $\mathbb{R}-$ Fuchsian structure $\rho_{0}$ of $\Sigma$, then for any simple closed geodesic $\alpha \in \mathbf{H}_{\mathbb{R}}^{2} / \Gamma_{0}, \Gamma_{0}=\rho_{0}\left(\pi_{1}\right)$, and for sufficiently small $t \in \mathbb{R}$ he shows the existence of a (continuous) bending deformation $\rho_{t}$ of $\rho_{0}$ induced by $\Gamma_{0}$-equivariant quasiconformal homeomorphisms $F_{t}$ of $\mathbf{H}_{\mathbb{C}}^{2}$. These homeomorphisms are extensions of Kourouniotis' bending homeomorphism to the complex hyperbolic space. Moreover, he shows that these deformations define an embedding of a $2 g-2$ ball into complex hyperbolic quasi-Fuchsian space $\mathcal{Q}_{\mathbb{C}}(\Sigma)$.

In this paper we choose to follow the strategy suggested in [2]. We fix a closed surface $\Sigma$ and an $\mathbb{R}$-Fuchsian, totally loxodromic and geometrically finite representation $\rho_{0}$ of $\pi_{1}(\Sigma)$. Then, $M_{0}=\mathbf{H}_{\mathbb{C}}^{2} / \Gamma_{0}$ is a complex hyperbolic manifold and embedded in $M_{0}$ there is a hyperbolic surface $\Sigma_{0}=\mathbf{H}_{\mathbb{R}}^{2} / \Gamma_{0}$. For every discrete geodesic lamination $\Lambda$ in $\Sigma$ with complex transverse measure $\mu$ and a simple closed geodesic $\alpha$ in $\Sigma_{0}$, we find an isometric map $B_{\mathbb{C}}$, depending on $\alpha$, of $M_{0}$ into a complex hyperbolic manifold $M_{h}$. (the complex hyperbolic quakebend map). Restricted to $\Sigma_{0}$ the image of this map is a pleated surface $\Sigma_{h}$, something which is entirely analogous to the classical case. The pleated surface $\Sigma_{h}$ is naturally embedded in $M_{h}$. Now Theorem A assures us that for small $t \in \mathbb{R}$, complex hyperbolic quakebending along $\Lambda$ with transverse measure $t \mu$ produces injective homomorphisms $\rho_{t \mu}$ of $\pi_{1}$ into the isometry group of $\mathbf{H}_{\mathbb{C}}^{2}$ with complex hyperbolic quasi-Fuchsian image. Our first main result is the following.

Theorem 3.09 For every $\mathbb{R}$-Fuchsian point $\rho_{0}\left(\pi_{1}\right)=\Gamma_{0}$ in $\mathcal{Q}_{\mathbb{C}}(\Sigma)$ and for every finite geodesic lamination with complex transverse measure $\mu$ in $\Sigma_{0}=\mathbf{H}_{\mathbb{R}}^{2} / \Gamma_{0}$, there is an $\epsilon>0$ such that for $|t|<\epsilon$ the complex hyperbolic quakebend curve $\rho_{t \mu}$ which is obtained by complex hyperbolic quakebending along $(\Lambda, \mu)$, lies entirely in $\mathcal{Q}_{\mathbb{C}}(\Sigma)$. 
We also note that $\rho_{t \mu}$ varies real analytically with $t$. Accordingly, we discuss the variations of the complex hyperbolic length $\lambda=l+i \theta$ of $\rho_{t \mu}$. The induced formulae are natural genaralisations of Epstein-Marden's formulae, although more complicated. For instance, for the first derivatives of $l=\Re(\lambda)$ and $\theta=\Im(\lambda)$ at $\rho_{0}$ we have the following ((4-6) and (4-7) of Theorem 4.4):

$$
\begin{aligned}
& \frac{d l\left(\rho_{t \mu}\right)}{d t}(0)=\sum_{k=1}^{n} \Re\left(\zeta_{k}\right) \cdot \cos \left(\phi_{k}\right), \\
& \frac{d \theta\left(\rho_{t \mu}\right)}{d t}(0)=\sum_{k=1}^{n} \Im\left(\zeta_{k}\right) \cdot \frac{3 \cos ^{2}\left(\phi_{k}\right)-1}{2} .
\end{aligned}
$$

Again, $\zeta_{k}=\mu\left(\alpha \cap \gamma_{k}\right)$ and $\phi_{k}$ is the oriented angle of intersection of $\alpha$ and $\gamma_{k}$. Note that the first equation is completely analogous to the formula (1-2). The second equation has no analogue in the classical case. We also give formulae for the second derivative of $\lambda\left(\rho_{t \mu}\right)$, see the formulae in Theorem 4.5. We only note here that in the case where $\mu$ is real, the formula for the second derivative of $l\left(\rho_{t \mu}\right)$ is exactly the same as in [2]. We finally remark that our results do not clarify if infinitesimal generators $t_{\mu}$ of the complex hyperbolic bending curves may define vector fields which span the tangent space of $\mathcal{Q}_{\mathbb{C}}(\Sigma)$ at $\rho_{0}$. This will be the subject of a forthcoming paper.

This paper is organised as follows. In the Section 2, we review in brief the Siegel domain model for complex hyperbolic space, its isometries and its totally geodesic submanifolds. We discuss loxodromic elements in Section 2.4, the trace and the complex hyperbolic length function in Section 2.5 and we go into some detail in discussing packs in Section 2.6. The main part of this paper lies in Section 3 and Section 4. In Section 3 , we construct the complex hyperbolic quakebending cocycle in subSection 3.2 and we examine its geometrical meaning. The complex hyperbolic quakebend map and the complex hyperbolic quakebend homomorphism and their properties are presented in Section 3.3 and Section 3.4 respectively. Our main Theorem 3.9 is at the end of this Section. Finally, Section 4 is devoted to the extensions of Wolpert-Kerckhoff formulae, namely Theorem 4.4 and Theorem 4.5 given in Section 4.2. The proof of these Theorems is preceded by the rather extensive preparatory Section 4.1 which includes several calculations.

\section{Acknowledgements}

The author wishes to thank JR Parker for reading this paper and making several useful suggestions. He also acknowledges support by a Marie Curie Reintegration Grant fellowship (Contract No. MERG-CT-2005-028371) within the 6th Community Framework Programme. 


\section{Preliminaries}

\subsection{Complex hyperbolic space}

In what follows we describe in brief the Siegel domain model for complex hyperbolic space; for further details the reader should consult for instance Goldman [4].

By $\mathbb{C}^{2,1}$ we denote the vector space $\mathbb{C}^{3}$ endowed with the $(2,1)$-Hermitian product $\langle\cdot, \cdot\rangle$ given by

$$
\langle\mathbf{z}, \mathbf{w}\rangle=z_{1} \bar{w}_{3}+z_{2} \bar{w}_{2}+z_{3} \bar{w}_{1} .
$$

We consider the subspaces

$$
\begin{aligned}
& V_{-}=\left\{\mathbf{z} \in \mathbb{C}^{2,1}:\langle\mathbf{z}, \mathbf{z}\rangle<0\right\}, \\
& V_{0}=\left\{\mathbf{z} \in \mathbb{C}^{2,1}-\{\mathbf{0}\}:\langle\mathbf{z}, \mathbf{z}\rangle=0\right\}
\end{aligned}
$$

and the canonical projection $\mathbb{P}: \mathbb{C}^{2,1}-\{0\} \longrightarrow \mathbb{C} P^{2}$ onto complex projective space. Complex hyperbolic space $\mathbf{H}_{\mathbb{C}}^{2}$ is defined to be $\mathbb{P}\left(V_{-}\right)$and its boundary $\partial \mathbf{H}_{\mathbb{C}}^{2}$ is $\mathbb{P}\left(V_{0}\right)$. It turns out that we can write $\mathbf{H}_{\mathbb{C}}^{2}=\mathbb{P}\left(V_{-}\right)$as

$$
\mathbf{H}_{\mathbb{C}}^{2}=\left\{\left(z_{1}, z_{2}\right) \in \mathbb{C}^{2}: 2 \Re\left(z_{1}\right)+\left|z_{2}\right|^{2}<0\right\}
$$

and also, for $\partial \mathbf{H}_{\mathbb{C}}^{2}=\mathbb{P}\left(V_{0}\right)$ we have

$$
\partial \mathbf{H}_{\mathbb{C}}^{2}-\{\infty\}=\left\{\left(z_{1}, z_{2}\right) \in \mathbb{C}^{2}: 2 \Re\left(z_{1}\right)+\left|z_{2}\right|^{2}=0\right\} .
$$

Given a point $z$ of $\mathbb{C}^{2} \subset \mathbb{C} P^{2}$ we may lift $z=\left(z_{1}, z_{2}\right)$ to a point $\mathbf{z}$ in $\mathbb{C}^{2,1}$, called the standard lift of $z$, by writing $\mathbf{z}$ in non-homogeneous coordinates as

$$
\mathbf{z}=\left[\begin{array}{c}
z_{1} \\
z_{2} \\
1
\end{array}\right] .
$$

We distinguish the following two points of $V_{0}$ :

$$
\mathbf{o}=\left[\begin{array}{l}
0 \\
0 \\
1
\end{array}\right], \quad \infty=\left[\begin{array}{l}
1 \\
0 \\
0
\end{array}\right] .
$$

These are the standard lifts of $o=(0,0)$ and $\infty$ respectively.

Complex hyperbolic space is a 2-complex dimensional complex Kähler manifold. Its Kähler form is induced by the Bergman metric on $\mathbf{H}_{\mathbb{C}}^{2}$ which is defined by the distance 
function $\rho$ given by the formula

$$
\cosh ^{2}\left(\frac{\rho(z, w)}{2}\right)=\frac{\langle\mathbf{z}, \mathbf{w}\rangle\langle\mathbf{w}, \mathbf{z}\rangle}{\langle\mathbf{z}, \mathbf{z}\rangle\langle\mathbf{w}, \mathbf{w}\rangle}=\frac{|\langle\mathbf{z}, \mathbf{w}\rangle|^{2}}{|\mathbf{z}|^{2}|\mathbf{w}|^{2}}
$$

where $\mathbf{z}$ and $\mathbf{w}$ in $V_{-}$are the standard lifts of $z$ and $w$ in $\mathbf{H}_{\mathbb{C}}^{2}$ and $|\mathbf{z}|=\sqrt{-\langle\mathbf{z}, \mathbf{z}\rangle}$. Alternatively, the metric tensor of the Bergman metric is given by

$$
d s^{2}=-\frac{4}{\langle\mathbf{z}, \mathbf{z}\rangle^{2}} \operatorname{det}\left[\begin{array}{cc}
\langle\mathbf{z}, \mathbf{z}\rangle & \langle d \mathbf{z}, \mathbf{z}\rangle \\
\langle\mathbf{z}, d \mathbf{z}\rangle & \langle d \mathbf{z}, d \mathbf{z}\rangle
\end{array}\right] .
$$

The holomorphic sectional curvature of $\mathbf{H}_{\mathbb{C}}^{2}$ equals to -1 and its real sectional curvature is pinched between -1 and $-1 / 4$.

\subsection{Isometries}

Denote by $\mathrm{U}(2,1)$ be the group of unitary matrices for the Hermitian product $\langle\cdot, \cdot\rangle$. The full group of holomorphic isometries of $\mathbf{H}_{\mathbb{C}}^{2}$ is $\mathrm{PU}(2,1)=\mathrm{U}(2,1) / \mathrm{U}(1)$, where $\mathrm{U}(1)=\left\{e^{i \theta} I, \theta \in[0,2 \pi)\right\}$ and $I$ is the $3 \times 3$ identity matrix. In this work we prefer to consider instead the group $\mathrm{SU}(2,1)$ of matrices which are unitary with respect to $\langle\cdot, \cdot\rangle$, and have determinant 1 . The group $\mathrm{SU}(2,1)$ is a 3 -fold covering of $\operatorname{PU}(2,1)$, a direct analogue of the fact that $\operatorname{SL}(2, \mathbb{C})$ is the double cover of $\operatorname{PSL}(2, \mathbb{C})$.

There exist three kinds of holomorphic isometries of $\mathbf{H}_{\mathbb{C}}^{2}$.

(i) Loxodromic isometries, each of which fixes exactly two points of $\partial \mathbf{H}_{\mathbb{C}}^{2}$. One of these points is attracting and the other repelling.

(ii) Parabolic isometries, each of which fixes exactly one point of $\partial \mathbf{H}_{\mathbb{C}}^{2}$.

(iii) Elliptic isometries, each of which fixes at least one point of $\mathbf{H}_{\mathbb{C}}^{2}$.

\subsection{Totally geodesic submanifolds}

Totally geodesic submanifolds of $\mathbf{H}_{\mathbb{C}}^{2}$ are always of codimension greater or equal than 2. The codimension 2 submanifolds come in two flavours. In the first place there are complex lines $L$, which have constant curvature -1 . These submanifolds realise isometric embeddings of $\mathbf{H}_{\mathbb{C}}^{1}$ (that is the hyperbolic plane with its complex structure) into $\mathbf{H}_{\mathbb{C}}^{2}$. Every complex line $L$ is the image under some $A \in \mathrm{SU}(2,1)$ of the complex line

$$
L_{0}=\left\{\left(z_{1}, z_{2}\right) \in \mathbf{H}_{\mathbb{C}}^{2}: z_{2}=0\right\} .
$$

The subgroup of $\mathrm{SU}(2,1)$ stabilising $L_{0}$ is thus conjugate to the group $\mathrm{S}(\mathrm{U}(1) \times$ $\mathrm{U}(1,1))<\mathrm{SU}(2,1)$. 
Secondly, we have totally real Lagrangian planes $R$ which have constant curvature $-1 / 4$. These in turn realise isometric embeddings of $\mathbf{H}_{\mathbb{R}}^{2}$ (that is the Klein-Beltrami model for hyperbolic plane) into $\mathbf{H}_{\mathbb{C}}^{2}$. Every Lagrangian plane is the image under some element of $\mathrm{SU}(2,1)$ of the standard real Lagrangian plane

$$
R_{\mathbb{R}}=\left\{\left(z_{1}, z_{2}\right) \in \mathbf{H}_{\mathbb{C}}^{2}: z_{i}=x_{i} \in \mathbb{R}, 2 x_{1}+x_{2}^{2}<0\right\} .
$$

This plane is preserved by $\mathrm{SO}(2,1)$, that is the subgroup of $\mathrm{SU}(2,1)$ comprising matrices with real entries.

Unlike the real hyperbolic space case, there are no totally geodesic submanifolds of codimension 1. But there exist fair substitutes, a class of which we shall describe below, see Section 2.6.

\subsection{Loxodromic isometries}

Let $A \in \mathrm{SU}(2,1)$ be a matrix representing a loxodromic isometry and consider its attractive fixed point. Associated to this point is an eigenvalue $e^{\lambda}$ of the matrix $A$ such as $\left|e^{\lambda}\right|=e^{\Re(\lambda)}>1$, that is $\Re(\lambda)>0$. It can be shown that the other two eigenvalues of $A$ are $e^{-\bar{\lambda}}$ (which is associated to the repelling fixed point of $A$ ) and $e^{\bar{\lambda}-\lambda}$. [4, Lemma 6.2.5]. We may also assume that $\Im(\lambda) \in(-\pi, \pi]$ and, in this way, $\lambda \in S$ where

$$
S=\{\lambda \in \mathbb{C}: \Re(\lambda)>0, \quad \Im(\lambda) \in(-\pi, \pi]\} .
$$

Let $a, r \in \partial \mathbf{H}_{\mathbb{C}}^{2}$ be the attractive and the repelling fixed points of $A$ respectively. Any lifts $\mathbf{a}, \mathbf{r}$ of $a, r$ to $V_{0}$ are eigenvectors of the matrix $A$ with corresponding eigenvalues $e^{\lambda}, e^{-\bar{\lambda}}$, where $\lambda \in S$. The geodesic $\alpha=(r, a)$ joining $r$ and $a$ is called the real axis of $A$. The fixed points $a$ and $r$ also span a complex line $L_{\alpha}$ in $\mathbf{H}_{\mathbb{C}}^{2}$, called the complex axis of $A$. The eigenvector $\mathbf{n}$ of $A$ corresponding to $e^{\bar{\lambda}-\lambda}$ is a polar vector to the complex axis $L_{\alpha}$.

For any $\lambda \in \mathbb{C}^{*}=\{\lambda \in \mathbb{C}:-\pi<\Im(\lambda) \leq \pi$ we define $E(\lambda) \in \mathrm{SU}(2,1)$ by

$$
E(\lambda)=\left[\begin{array}{ccc}
e^{\lambda} & 0 & 0 \\
0 & e^{\bar{\lambda}-\lambda} & 0 \\
0 & 0 & e^{-\bar{\lambda}}
\end{array}\right] .
$$

If $\lambda \in S$ then $E=E(\lambda)$ is a loxodromic map with attractive eigenvalue $e^{\lambda}$ and attractive (resp. repelling) fixed point $\infty$ (resp. $o$ ). If $\Re(\lambda)=0$ then $E(\lambda)$ is elliptic (or the identity) and fixes the complex line spanned by $o$ and $\infty$. If $\Re(\lambda)<0$ then 
$-\bar{\lambda} \in S$ and $E(\lambda)$ is a loxodromic map with attractive fixed point $o$ and repelling fixed point $\infty$.

Let $A$ be a general loxodromic map with attracting eigenvalue $e^{\lambda}$ for $\lambda \in S$. Since $\mathrm{SU}(2,1)$ acts 2 -transitively on $\partial \mathbf{H}_{\mathbb{C}}^{2}$ then there exists a $Q \in \mathrm{SU}(2,1)$ whose columns are projectively $\mathbf{a}, \mathbf{n}, \mathbf{r}$. Moreover, $\mathbf{a}=Q(\infty)$ and $\mathbf{r}=Q(\mathbf{o})$. Thus we may write:

$$
A=Q E(\lambda) Q^{-1}
$$

where $E(\lambda)$ is given by (2-2).

If $A$ lies in $\mathrm{SO}(2,1)$ and corresponds to a loxodromic isometry of the hyperbolic plane then $\lambda$ is real and so $\operatorname{tr}(A)=2 \cosh (\lambda)+1$ is real and greater than 3 . If $\Im(\lambda)=\pi$ then $A$ corresponds to a hyperbolic glide reflection on $\mathbf{H}_{\mathbb{R}}^{2}$ and $\operatorname{tr}(A)=-2 \cosh (\Re(\lambda))+1<$ -1 .

\subsection{Trace and complex hyperbolic length}

Let $A$ be a loxodromic matrix with eigenvalues $e^{\lambda}, e^{\bar{\lambda}-\lambda}, e^{-\bar{\lambda}}$ where we suppose that $e^{\lambda}$ is its attractive eigenvalue and therefore $\lambda \in S$. The trace of $A$ is given by the following function of $\lambda$ which we denote by $\tau(\lambda)$ :

$$
\operatorname{tr}(A)=\tau(\lambda)=e^{\lambda}+e^{\bar{\lambda}-\lambda}+e^{-\bar{\lambda}}
$$

The following is in Parker-Platis [11].

Lemma 2.1 The function $\tau(\lambda)=e^{\lambda}+e^{\bar{\lambda}-\lambda}+e^{-\bar{\lambda}}$ is a real analytic diffeomorphism from $S$ onto $T$, where $T$ is the exterior of the deltoid curve $\delta(t)=2 e^{i t}+e^{-2 i t}$, $t \in[0,2 \pi)$.

Definition 2.2 The inverse $\lambda: T \rightarrow S$ of $\tau$ shall be called the complex hyperbolic length function.

If $A \in \mathrm{SU}(2,1)$ is loxodromic, then $\exp (\lambda(\operatorname{tr}(A)))$ is the attractive eigenvalue of $A$. The number $\lambda(A)$ is called the complex hyperbolic length of $A$. Its real part $l(A)=\Re(\lambda(A))$ is half the geodesic length of the real axis $\alpha$ of $A$ and $\theta(A)=\Im(\lambda(A))$ is half the rotation angle about $\alpha$. 


\subsection{Packs}

As we have mentioned above, there are no totally geodesic hypersurfaces of complex hyperbolic space. There are two classes of substitutes, namely bisectors and packs. Bisectors will not concern us here; the interested reader should consult [4] for an extensive presentation of bisectors. Packs are the counterpart of bisectors: in general, a pack is real analytic 3-dimensional submanifold of complex hyperbolic space which is naturally foliated by Lagrangian planes. In what follows we shall review briefly the definition of a pack given in the most general setting in [10]. A weaker definition given by P Will may be found in [17] as well as in [3].

Let $A=Q E(\lambda) Q^{-1}$ be a loxodromic map as in (2-3). For any $x \in \mathbb{R}$ define $A^{x}$ by

$$
A^{x}=Q E\left(\lambda^{x}\right) Q^{-1} .
$$

The transformation $A^{x}$ has the same eigenvectors as $A$, but its eigenvalues are the eigenvalues of $A$ raised to the $x$ th power. Hence we immediately see that $A^{x}$ is a loxodromic element of $\mathrm{SU}(2,1)$ for all $x \in \mathbb{R}-\{0\}$ and $A^{0}=I$. Moreover, for any integer $n, A^{n}$ agrees with the usual notion of the $n$th power of $A$. The following is proved in [10, Proposition 3.1].

Proposition 2.3 Let $R_{0}$ and $R_{1}$ be disjoint Lagrangian planes in $\mathbf{H}_{\mathbb{C}}^{2}$ and let $\iota_{0}$ and $\iota_{1}$ be the respective inversions. Consider the loxodromic map $A=\iota_{1} \iota_{0}$ and its powers $A^{x}$ for each $x \in \mathbb{R}$. Then:

(i) $\iota_{x}$ defined by $A^{x}=\iota_{x} \iota_{0}$ is inversion in a Lagrangian plane $R_{x}=A^{x / 2}\left(R_{0}\right)$.

(ii) $R_{x}$ intersects the complex axis $L_{A}$ of $A$ orthogonally in a geodesic $\gamma_{x}$.

(iii) The geodesics $\gamma_{x}$ are the leaves of a foliation of $L_{A}$.

(iv) For each $x \neq y \in \mathbb{R}, R_{x}$ and $R_{y}$ are disjoint.

Definition 2.4 Given disjoint Lagrangian planes $R_{0}$ and $R_{1}$, then for each $x \in \mathbb{R}$ let $R_{x}$ be the Lagrangian plane constructed in Proposition 2.3. Define

$$
P=P\left(R_{0}, R_{1}\right)=\bigcup_{x \in \mathbb{R}} R_{x} .
$$

We call $P$ the pack determined by $R_{0}$ and $R_{1}$.

The set $P$ is a real analytic 3 -submanifold of $\mathbf{H}_{\mathbb{C}}^{2}$. We call $\gamma=\operatorname{Ax}\left(\iota_{1} \iota_{0}\right)$ the spine of $P$ and the Lagrangian planes $R_{x}$ for $x \in \mathbb{R}$ the slices of $P$. Moreover, by [10, Proposition 3.3], $P$ is homeomorphic to a 3-ball whose boundary lies in $\partial \mathbf{H}_{\mathbb{C}}^{2}$ and also the complement $\mathbf{H}_{\mathbb{C}}^{2}-P$ of $P$, has two components, each homeomorphic to a 
4-ball. Observe that $P$ contains $L$, the complex line containing $\gamma$, the spine of $P$. The boundary of $P$ contains the boundary of the complex line $L$ and is foliated by the boundaries of the Lagrangian planes $R_{x}$.

From a Lagrangian plane $R$ and a geodesic $\gamma \in \mathbb{R}$ we may construct a pack according to the following ([10, Proposition 3.4]).

Proposition 2.5 Suppose that the geodesic $\gamma$ lies on a Lagrangian plane $R$. Then the set

$$
P(\gamma)=\Pi_{R}^{-1}(\gamma)=\bigcup_{z \in \gamma} \Pi_{R}^{-1}(z)
$$

is the pack determined by the Lagrangian planes $R_{0}=\Pi_{R}^{-1}\left(z_{0}\right)$ and $R_{1}=\Pi_{R}^{-1}\left(z_{1}\right)$ for any distinct points $z_{0}, z_{1} \in \gamma$. Moreover, for each $z \in \gamma$, the Lagrangian plane $\Pi_{R}^{-1}(z)$ is a slice of $P(\gamma)$.

\section{Complex hyperbolic quakebending of $\mathbb{R}$-Fuchsian struc- tures}

Let $\Sigma$ be a closed (that is compact and without boundary) topological surface of genus $g>1$ and denote by $\pi_{1}=\pi_{1}(\Sigma)$ its fundamental group. A complex hyperbolic quasi-Fuchsian representation is a homomorphism $\rho: \pi_{1} \rightarrow \mathrm{SU}(2,1)$ which is discrete, faithful, totally loxodromic and geometrically finite. We should make two remarks here (see also [10, Section 1] and the references therein). In the first place, if a representation $\rho: \pi_{1} \rightarrow \mathrm{SU}(2,1)$ is totally loxodromic and its image neither fixes a point at the boundary of $\mathbf{H}_{\mathbb{C}}^{2}$ nor preserves a totally geodesic subspace, then it is automatically discrete. Moreover, geometrical finiteness here is in the sense of Bowditch: A discrete subgroup $\Gamma$ of $\operatorname{SU}(2,1)$ with region of discontinuity $\Omega \subset \partial \mathbf{H}_{\mathbb{C}}^{2}$ has the property F1, that is $\Gamma$ is geometrically finite in the first sense, if the orbifold $M=\left(\mathbf{H}_{\mathbb{C}}^{2} \cup \Omega\right) / \Gamma$ has only finitely many topological ends, each of which is a parabolic end.

Definition 3.1 The complex hyperbolic quasi-Fuchsian space $\mathcal{Q}_{\mathbb{C}}=\mathcal{Q}_{\mathbb{C}}(\Sigma)$ of $\Sigma$ is the quotient of the space $\operatorname{Hom}\left(\pi_{1} \rightarrow \mathrm{SU}(2,1)\right)$ of complex hyperbolic quasiFuchsian representations $\rho$ of $\pi_{1}$ into $\operatorname{SU}(2,1)$ modulo the left action of $\operatorname{SU}(2,1)$ on $\operatorname{Hom}\left(\pi_{1} \rightarrow \mathrm{SU}(2,1)\right)$ by inner automorphisms.

According to Theorem A, if $\rho_{0}: \pi_{1} \rightarrow \mathrm{SU}(2,1)$ is an $\mathbb{R}$-Fuchsian representation of $\pi_{1}$ then there exists an open neighbourhood $U=U\left(\rho_{0}\right)$ of $\rho_{0}$ in $\operatorname{Hom}\left(\pi_{1} \rightarrow\right.$ $\mathrm{SU}(2,1)) / \mathrm{SU}(2,1)$ so that any representation $\rho$ in $U$ is complex hyperbolic quasiFuchsian. Having this in hand, we now proceed to the setting of our construction. 


\subsection{The setting}

We fix an $\mathbb{R}$-Fuchsian point $\rho_{0} \in \mathcal{Q}_{\mathbb{C}}$ and we may conjugate so that $\rho_{0}\left(\pi_{1}\right)=\Gamma_{0}$ is a subgroup of $\mathrm{SO}(2,1)$, that is $\Gamma_{0}$ fixes the standard real Lagrangian plane $R_{\mathbb{R}}$. Then the quotient $M_{0}=\mathbf{H}_{\mathbb{C}}^{2} / \Gamma_{0}$ is a complex hyperbolic quasi-Fuchsian manifold and embedded in $M_{0}$ is the 2-dimensional manifold $\Sigma_{0}=\mathbf{H}_{\mathbb{R}}^{2} / \Gamma_{0}$ which carries a hyperbolic structure inherited by the complex hyperbolic structure of $M_{0}$. From now on we identify the Lagrangian plane $R_{\mathbb{R}}$ to $\mathbf{H}_{\mathbb{R}}^{2}$ and we agree that an orientation on $\mathbf{H}_{\mathbb{R}}^{2}$ is fixed.

Let the pair $(\Lambda, \mu)$ denote a discrete complex measured geodesic lamination $\Lambda$ of $\Sigma_{0}$ with transverse measure $\mu$ and also denote by $(\widetilde{\Lambda}, \tilde{\mu})$ its lift to $\mathbf{H}_{\mathbb{R}}^{2}$. The action of $\Gamma_{0}$ in $\mathbf{H}_{\mathbb{R}}^{2}$ leaves $(\widetilde{\Lambda}, \tilde{\mu})$ invariant, ie for every $A \in \Gamma_{0}, A(\widetilde{\Lambda})=\widetilde{\Lambda}$ and $A_{*} \tilde{\mu}=\tilde{\mu}$.

We shall hereafter suppose that $\Lambda$ (and therefore $\widetilde{\Lambda}$ ) is finite and thus discrete. The study of the case where $\Lambda$ is infinite goes beyond the scope of this work and will not concern us here.

\subsection{Complex hyperbolic quakebend cocycle}

Definition 3.2 A $\rho_{0}$-cocycle is a map

$$
C: \mathbf{H}_{\mathbb{R}}^{2} \times \mathbf{H}_{\mathbb{R}}^{2} \rightarrow \operatorname{SU}(2,1)
$$

such that:

(1) if $x \in \mathbf{H}_{\mathbb{R}}^{2}$, then $C(x, x)=I$, the identity element of $\mathrm{SU}(2,1)$,

(2) if $x, y, z \in \mathbf{H}_{\mathbb{R}}^{2}$, then $C(x, y) C(y, z)=C(x, z)$ and

(3) for every $x, y \in \mathbf{H}_{\mathbb{R}}^{2}$ and $A=\rho_{0}(a) \in \Gamma_{0}, a \in \pi_{1}$ we have

$$
C(A x, A y)=A C(x, y) A^{-1} .
$$

Remark 3.3 Conditions (1) and (2) imply $C(x, y)=C(y, x)^{-1}$.

Remark 3.4 From a $\rho_{0}$-cocycle we may define a homomorphism $\rho_{C}: \pi_{1} \rightarrow \operatorname{SU}(2,1)$ as follows. We fix a point $x \in \mathbf{H}_{\mathbb{R}}^{2}$ and put

$$
C(A)=C(x, A x), \quad A=\rho_{0}(a) \in \Gamma_{0}, \quad a \in \pi_{1} .
$$

Then $\rho_{C}$ is defined by the relation

$$
\rho_{C}(a)=C\left(\rho_{0}(a)\right) \rho_{0}(a), \quad a \in \pi_{1} .
$$


Indeed, for every $a, b \in \pi_{1}$ such that $\rho_{0}(a)=A$ and $\rho_{0}(b)=B$ we have

$$
\begin{aligned}
C\left(\rho_{0}(a), \rho_{0}(b)\right)=C(A, B) & =C(x, A B x) \\
& =C(x, A x) C(A x, A B x) \\
& =C(A) A C(x, B x) A^{-1} \\
& =C(A) A C(B) A^{-1} \\
& =C\left(\rho_{0}(a)\right) \rho_{0}(a) C\left(\rho_{0}(b)\right)\left(\rho_{0}(a)\right)^{-1},
\end{aligned}
$$

where we have used (2) to obtain the first equality and (3) to obtain the penultimate equality.

3.2.1 Construction of quakebend cocycle Let $(\Lambda, \mu)$ be a finite complex measured geodesic lamination on $\Sigma_{0}=\mathbf{H}_{\mathbb{R}}^{2} / \Gamma_{0}$ and as before, denote by $(\widetilde{\Lambda}, \tilde{\mu})$ its lift to $\mathbf{H}_{\mathbb{R}}^{2}$. We are going to construct a special $\rho_{0}$-cocycle $B$ (associated to $\left.\Lambda, \mu\right)$ ) which we shall call the complex hyperbolic quakebend cocycle. For this, consider two arbitrary points $x, y \in \mathbf{H}_{\mathbb{R}}^{2}$ and make the following assumptions.

(1) If both $x, y$ belong to the same component of the complement of $\widetilde{\Lambda}$, then we set $B(x, y)=I$, the identity element of $\mathrm{SU}(2,1)$.

(2) If not, then denote by $[x, y]$ the oriented from $x$ to $y$ closed geodesic segment and number the leaves which intersect $[x, y]$ starting from $x$, say $\gamma_{1}, \ldots, \gamma_{n}$. We orient each $\gamma_{k}$ so that it crosses $[x, y]$ from the right to the left and let $\zeta_{k}=\tilde{\mu}\left(\gamma_{k} \cap[x, y]\right)$.

Let $\alpha$ be the oriented geodesic $(0, \infty)$ and $\gamma=(p, q)$ be any oriented geodesic of $\mathbf{H}_{\mathbb{R}}^{2}, S$ be the domain defined in $(2-1)$ and $\zeta \in S \cup(-S)$ be a complex number. To the pair $(\gamma, \zeta)$ we associate a loxodromic element $E(\gamma, \zeta)$ of $\mathrm{SU}(2,1)$ which is conjugate in $\mathrm{SU}(2,1)$ to

$$
E(\alpha, \zeta)=\left[\begin{array}{ccc}
e^{\zeta} & 0 & 0 \\
0 & e^{\bar{\zeta}-\zeta} & 0 \\
0 & 0 & e^{-\bar{\zeta}}
\end{array}\right] .
$$

Following the discussion in Section 2.4,

$$
E(\gamma, \zeta)=Q E(\alpha, \zeta) Q^{-1}
$$

where $Q$ is given here in the following way. If $\mathbf{p}=\left[\begin{array}{lll}p_{1} & p_{2} & p_{3}\end{array}\right]^{T}$ and $\mathbf{q}=\left[\begin{array}{lll}q_{1} & q_{2} & q_{3}\end{array}\right]^{T}$ are lifts to $V_{0}$, then the polar vector to $\mathbf{p}$ and $\mathbf{q}$ is

$$
\mathbf{n}=\left[\begin{array}{lll}
p_{2} q_{3}-p_{3} q_{2} & p_{3} q_{1}-p_{1} q_{3} & p_{2} q_{1}-p_{1} q_{2}
\end{array}\right]^{T} .
$$


We may choose $\mathbf{p}$ and $\mathbf{q}$ so that $\langle\mathbf{p}, \mathbf{q}\rangle=1$; then $\langle\mathbf{n}, \mathbf{n}\rangle=1$ and $Q=[\mathbf{q} \mathbf{n} \mathbf{p}] \in$ $\mathrm{SU}(2,1)$. In this way, if $\zeta \in S$ then $E(\gamma, \zeta)$ is a loxodromic element of $\mathrm{SU}(2,1)$ with complex hyperbolic length $\zeta$ and real axis $\gamma$. If $\zeta \in-S$ then, $E(\gamma, \zeta)$ is a loxodromic element of $\mathrm{SU}(2,1)$ with complex hyperbolic length $-\zeta$ and real axis $-\gamma$.

Now for $k=1, \ldots, n$ we set

$$
B_{k}=E\left(\gamma_{k}, \zeta_{k}\right)
$$

where we may have to replace $\zeta_{1}$ (resp. $\zeta_{n}$ ) by $\zeta_{1} / 2$ (resp. by $\zeta_{n} / 2$ ) if $\gamma_{1}$ passes through $x$ (resp. if $\gamma_{n}$ passes through $y$ ). We then define

$$
B(x, y)=\prod_{k=1}^{n} B_{k}
$$

Proposition 3.5 $B$ is a $\rho_{0}$-cocycle on $\mathbf{H}_{\mathbb{R}}^{2}$.

Proof The proof follows the lines of Kourouniotis [8, Lemma 2.1]. By definition $B(x, x)=I$ for all $x \in \mathbf{H}_{\mathbb{R}}^{2}$. Now let $x, y, z$ be arbitrary points and assume first that $y \in[x, z]$ and is not lying on any leaf of $\widetilde{\Lambda}$. Suppose that $\gamma_{1}, \ldots, \gamma_{l}$ are the leaves which intersect $[x, y]$ and $\gamma_{l+1}, \ldots, \gamma_{m}$ are these which intersect $[y, z]$. Then

$$
B(x, y) B(y, z)=\prod_{k=1}^{l} B_{k} \prod_{k=1}^{m-l-1} B_{l+k+1}=\prod_{k=1}^{m} B_{k}=B(x, z) .
$$

Suppose now that $y$ lies on some leaf $\gamma_{l}$ which intersects $[x, z]$. Then

$$
B(x, y) B(y, z)=\left(\prod_{k=1}^{l-1} B_{k} \cdot E\left(\gamma_{l}, \zeta_{l} / 2\right)\right) \cdot\left(E\left(\gamma_{l}, \zeta_{l} / 2\right) \cdot \prod_{k=1}^{m-l-1} B_{l+k+1}\right)=B(x, z) .
$$

The same calculations hold when $y$ lies on a component of $\mathbf{H}_{\mathbb{R}}^{2}-\widetilde{\Lambda}$ which intersects $[x, z]$. In all other cases there are points $s_{1} \in[y, z], s_{2} \in[x, z]$ and $s_{3} \in[x, y]$ such that $s_{1}$ separates the leaves that intersect $[x, y]$ and $[y, z]$ from those which intersect $[y, z]$ and $[x, z]$ and similarly for $s_{2}$ and $s_{3}$. Therefore,

$$
\begin{aligned}
B(x, z) & =B C\left(x, s_{2}\right) B\left(s_{2}, z\right) \\
& =B\left(x, s_{3}\right) B\left(s_{1}, z\right) \\
& =B\left(x, s_{3}\right) B\left(s_{3}, y\right) B\left(y, s_{1}\right) B\left(s_{1}, z\right) \\
& =B(x, y) B(y, z) .
\end{aligned}
$$

Finally, we prove condition (3) of Definition 3.2. Since $(\widetilde{\Lambda}, \tilde{\mu})$ is invariant under $\Gamma_{0}$, we have that for each $A=\rho_{0}(a) \in \Gamma_{0}$ the leaves which intersect the closed 
segment $[A x, A y]$ are just $A\left(\gamma_{0}\right), \ldots, A\left(\gamma_{n}\right)$ and for every $x_{k} \in \gamma_{k}, \tilde{\mu}\left(\left\{A\left(x_{k}\right)\right\}\right)=\zeta_{k}$. If $p_{k}, q_{k}$ are the endpoints of $\gamma_{k}$, then $E\left(A\left(\gamma_{k}\right), \zeta_{k}\right)$ has endpoints $A\left(p_{k}\right), A\left(q_{k}\right)$ respectively. Now the transformations $E\left(A\left(\gamma_{k}\right), \zeta_{k}\right)$ and $A E\left(\gamma_{k}, \zeta_{k}\right) A^{-1}$ have the same fixed points and the same complex length and thus they are equal. This completes the proof.

Before we go on to define explicitly the complex hyperbolic quakebending map in the next section, we wish at this point to discuss in some detail the geometric meaning of the complex hyperbolic quakebend cocycle $B=\prod_{k=1}^{n} B_{k}, B_{k}=E\left(\gamma_{k}, \zeta_{k}\right)$. In this way, the connection of complex hyperbolic bending with the classical case will be transparent.

Working in universal covers, let as before $\widetilde{\Lambda}=\bigcup_{k=1}^{n} \gamma_{k}$ be our bending lamination. The domain $R_{\mathbb{R}}-\widetilde{\Lambda}$ has $n+1$ components which we number from the left to the right as $S_{0}, S_{1}, \ldots, S_{n}$. We denote by $P_{k}$ the packs $P\left(\gamma_{k}\right)$ and by $\mathcal{S}_{k}$ we denote the flat pieces $\Pi^{-1}\left(S_{k}\right), k=0, \ldots, n$ where as usual, $\Pi$ is the orthogonal projection to the standard real Lagrangian plane. These flat pieces are the $n+1$ components of the domain $\mathbf{H}_{\mathbb{C}}^{2}-\bigcup_{k=1}^{n} P\left(\gamma_{k}\right)$.

In the first step of bending, the transformation $B_{n}=E\left(\gamma_{n}, \zeta_{n}\right)$ maps $P\left(\gamma_{n}\right)$ to the pack $B_{n}\left(P\left(\gamma_{n}\right)\right)$ in a way such that $\gamma_{n}$ is mapped to itself. Therefore, this pack has the same real spine as $P\left(\gamma_{n}\right)$, that is $\gamma_{n}$, but now it is considered to lie on the Lagrangian plane $B_{n}\left(R_{\mathbb{R}}\right)$ which is inclined to $R_{\mathbb{R}}$ in an angle $2 \Im\left(\zeta_{n}\right)$. Furthermore, the sector $\mathcal{S}_{n}$ is mapped to the sector $B_{n}\left(\mathcal{S}_{n}\right)$ in a way such its "basis" $B_{n}\left(S_{n}\right)$ lies on $B_{n}\left(R_{\mathbb{R}}\right)$.

In the second step, the transformation $B_{n-1}$ is applied to $\mathcal{S}_{n-1} \cup B_{n}\left(\mathcal{S}_{n}\right)$. The pack $P\left(\gamma_{n}\right)$ is now mapped to the pack $B_{n-1}\left(P\left(\gamma_{n}\right)\right)$. This pack has real spine $B_{n-1}\left(\gamma_{n}\right)$ lying on the Lagrangian plane $B_{n-1}\left(R_{\mathbb{R}}\right)$ which is inclined to $R_{\mathbb{R}}$ in an angle $2 \Im\left(\zeta_{n-1}\right)$. The sector $B_{n-1}\left(\mathcal{S}_{n-1}\right)$ is thus based on $B_{n-1}\left(R_{\mathbb{R}}\right)$.

By exhausting the process we have on one hand the real geodesics

$$
\gamma_{1}, B_{1}\left(\gamma_{2}\right), \ldots, B_{1} \circ \cdots \circ B_{n-1}\left(\gamma_{n}\right)
$$

which are such that each successive pair $\left(B_{k-1}\left(\gamma_{k}\right), B_{k}\left(\gamma_{k+1}\right)\right)$ of geodesics bounds a 2-dimensional sector $S_{k}^{b}$ lying on a Lagrangian plane $R_{k}^{b}$, which is isometric to some $S_{k}$. Additionally, at the $k$ th geodesic the two adjacent sectors are inclined in an angle $2 \Im\left(\zeta_{k}\right)$ and moreover, for each $k$, the sector $S_{k}$ on the right of $\gamma_{k}$ slides a distance $2 \Re\left(\zeta_{k}\right)$ relative to the sector $S_{k-1}$ on its left. On $\gamma_{k}, b_{\mathbb{R}}$ slides half the distance compared to the sectors at each side.

Definition 3.6 We call the union of the real geodesics (3-1) and the sectors $S_{k}^{b}$, the pleated surface $\widetilde{\Sigma}_{b}$ induced by complex hyperbolic bending along $\tilde{\mu}$. 
Now on the other hand, the process of bending also determines the packs

$$
P\left(\gamma_{1}\right), B_{1}\left(P\left(\left(\gamma_{1}\right)\right)\right), \ldots, B_{1} \circ \cdots \circ B_{n-1}\left(P\left(\gamma_{n}\right)\right) .
$$

These are such that each successive pair $\left(B_{k-1}\left(P\left(\gamma_{k-1}\right)\right), B_{k+1}\left(P\left(\gamma_{k+1}\right)\right)\right)$ of packs bounds a 4-dimensional sector $\mathcal{S}_{k}^{b}=\Pi_{R_{k}^{b}}^{-1}\left(S_{k}^{b}\right)$ "based" on $S_{k}^{b}$, which is isometric to some $\mathcal{S}_{k}$. In this way, $\mathbf{H}_{\mathbb{C}}^{2}$ may be thought as the union of the sectors $\mathcal{S}_{k}^{b}$, such that each successive pair of them has common "edge" one of the packs of (3-2).

\subsection{The complex hyperbolic quakebend map}

Following the previous discussion, we now associate to the bending cocycle $B$ constructed for the finite lamination $(\widetilde{\Lambda}, \tilde{\mu})$, the complex hyperbolic quakebend map $b_{\mathbb{C}}: \mathbf{H}_{\mathbb{C}}^{2} \rightarrow \mathbf{H}_{\mathbb{C}}^{2}$. Its restriction $b_{\mathbb{R}}$ to $\mathbf{H}_{\mathbb{R}}^{2}$ is the accurate analogue of the quakebend map $h$ defined in [2]. Pick up a base point in $\mathbf{H}_{\mathbb{R}}^{2}$ say $O$, and denote by $\Pi: \mathbf{H}_{\mathbb{C}}^{2} \rightarrow R_{\mathbb{R}}$ the projection on the standard real Lagrangian plane. Then, for each $z \in \mathbf{H}_{\mathbb{C}}^{2}$

$$
b_{\mathbb{C}}(z)=B(O, \Pi(z))(z) .
$$

Obviously, for $x \in \mathbf{H}_{\mathbb{R}}^{2}$ we have

$$
b_{\mathbb{R}}(x)=B(O, x)(x) .
$$

Clearly, the definition of $b_{\mathbb{C}}$ depends on the choice of the base point. We summarise some of the properties of $b_{\mathbb{C}}$ in the following Proposition; its proof follows directly from the discussion of the previous section and the reader should compare with $[2$, Section 3.6].

Proposition 3.7 The complex hyperbolic quakebend map $b_{\mathbb{C}}$ enjoys the following properties.

(1) If $R=\Pi^{-1}(O)$, where $\Pi: \mathbf{H}_{\mathbb{C}}^{2} \rightarrow R_{\mathbb{R}}$ is the orthogonal projection, then $b_{\mathbb{C}}(R)=R$ point wise. Consequently, $b_{\mathbb{C}}(O)=O$.

(2) The restriction of $b_{\mathbb{C}}$ to each flat piece $\Pi^{-1}\left(S_{k}\right), k=0, \ldots, n$ is an isometry.

(3) $B_{\mathbb{C}}$ is continuous except at points $z \in \mathbf{H}_{\mathbb{C}}^{2}$ where $\Re(\tilde{\mu}(\{\Pi(z)\}))>0$.

We close this section by distinguishing two special cases.

(1) $\mu$ is purely imaginary. Then the quakebend cocycle consists only of rotations about the geodesics with bending angles $\Im\left(\zeta_{k}\right)$. The map $b_{\mathbb{C}}$ preserves real geodesic lengths and is called pure bending.

(2) $\quad \mu$ is pure real. Then $b_{\mathbb{R}}\left(R_{\mathbb{R}}\right)=R_{\mathbb{R}}$ but the geodesics $\gamma_{k}$ have been moved in a particular way. If $\Re\left(\zeta_{k}\right)>0$ (resp. $\Re\left(\zeta_{k}\right)<0$ ) then $b_{\mathbb{C}}$ is called a left (resp. right) earthquake. 


\subsection{The complex hyperbolic quakebending homomorphism}

By Remark 3.4 it follows that from the $\rho_{0}$-bending cocycle $B$ we may define a homomorphism $\rho_{B}: \pi_{1} \rightarrow \mathrm{SU}(2,1)$. We choose a base point $O \in \mathbf{H}_{\mathbb{R}}^{2}$ and for each $a \in \pi_{1}$ we set

$$
\rho_{B}(a)=B\left(O, \rho_{0}(a)(O)\right) \circ \rho_{0}(a) .
$$

We shall call $\rho_{B}$ the complex hyperbolic quakebending homomorphism associated to $\rho_{0}$.

Let $\rho_{0},(\Lambda, \mu)$ and $(\widetilde{\Lambda}, \tilde{\mu})$ as before and let also $t \in \mathbb{R}$. Pick up any two points $x, y \in \mathbf{H}_{\mathbb{R}}^{2}$ and consider $B_{t}(x, y)$, that is the bending $\rho_{0}$-cocycle formed with respect to $(\widetilde{\Lambda}, t \tilde{\mu})$.

Proposition 3.8 The following hold.

(1) $B_{t}(x, y)$ is a real analytic function of $t \in \mathbb{R}$.

(2) There is an $\varepsilon>0$ and a neighborhood $\Delta_{\varepsilon}=\{t \in \mathbb{R}:|t|<\varepsilon\}$ such that for each $t \in \Delta_{\varepsilon}$ the homomorphism

$$
\rho_{t}=\rho_{B_{t}}
$$

is injective.

Proof To prove (1) suppose that $[x, y]$ intersects $\widetilde{\Lambda}$ at the leaves $\gamma_{1}, \ldots, \gamma_{n}$ and that $x$ and $y$ do not belong to any of $\gamma_{0}$ and $\gamma_{n}$ respectively. Then $B_{t}(x, y)$ may be written as

$$
B_{t}(x, y)=\prod_{k=1}^{n} Q_{k} E\left(t \zeta_{k}\right) Q_{k}^{-1}
$$

where

$$
E\left(t \zeta_{k}\right)=\left[\begin{array}{ccc}
e^{t \zeta_{k}} & 0 & 0 \\
0 & e^{t\left(\overline{\zeta_{k}}-\zeta_{k}\right)} & 0 \\
0 & 0 & e^{-t \overline{\zeta_{k}}}
\end{array}\right] .
$$

In general, we may have to divide $\zeta_{1}=\tilde{\mu}\left(\gamma_{1}\right)$ or $\zeta_{n}=\tilde{\mu}\left(\gamma_{n}\right)$ by two, depending on whether $x, y$ belong to $\gamma_{1}$ and $\gamma_{n}$ respectively. In all cases the entries of $B_{t}(x, y)$ are clearly real analytic functions of $t$.

To prove (2) it suffices to show that except for at most countably infinite set of points in $\mathbb{R}$, the homomorphism $\rho_{t}$ is injective. For this, consider an element $A=\rho_{0}(a) \in \Gamma_{0}$ where $a$ is not the identity in $\pi_{1}$. Then, the function $\Re(\lambda)(t)=\Re\left(\lambda\left(\rho_{t}(a)\right)\right)$ does not take the value 3 at $t=0$. Thus, except perhaps for a discrete set of values of $t \in \mathbb{R}$, $\Re(\lambda)(t) \neq 3$ and therefore, $\rho_{t}(a) \neq I$. 
From Proposition 3.8 and Theorem A we immediately obtain our main theorem.

Theorem 3.9 For all $t$ as in (2) of Proposition 3.8,

$$
\left.\rho_{t} \in \operatorname{Hom}\left(\pi_{1} \rightarrow \mathrm{SU}(2,1)\right) / \mathrm{SU}(2,1)\right) .
$$

By taking a smaller $\epsilon>0$ if necessary, we additionally have $\rho_{t} \in \mathcal{Q}_{\mathbb{C}}$.

Combining Theorem 3.9 and the discussion at the end of Section 3.2 we conclude that quakebending along $t \mu, t \in \Delta_{\varepsilon}$, produces two geometrical objects: the one is a 2-dimensional hyperbolic surface $\Sigma_{b_{t}}$ isometric to $\Sigma_{0}$ and is the direct analogue of a pleated surface as in the classical case. The other is a 4-dimensional complex hyperbolic manifold $M_{b_{t}}=\mathbf{H}_{\mathbb{C}}^{2} / \Gamma_{t}, \Gamma_{t}=\rho_{B_{t}}\left(\pi_{1}\right)$, which is isometric to $M_{0}$ in its flat pieces and has $\Sigma_{b_{t}}$ as an embedded 2-submanifold.

\section{Derivatives}

In this section, we shall be concerned with the derivatives of the complex hyperbolic length function $\lambda(t)$ of the complex hyperbolic quakebend deformation $B_{t}$ associated to the measure $t \mu$ of a transformation $A=\rho_{0}(a), a \in \pi_{1}$. We need some preparation first.

\subsection{Derivatives of complex hyperbolic length}

Let $A$ be a loxodromic element of $\mathrm{SO}(2,1)$ and consider a deformation $F$ of $A$. That is a map

$$
F: \Delta_{\varepsilon} \rightarrow \mathrm{SU}(2,1)
$$

of a neighborhood $\Delta_{\varepsilon}=\{t \in \mathbb{R},|t|\langle\varepsilon, \varepsilon>0\}$ into $\mathrm{SU}(2,1)$ such that $F(0)=A$. Let $\tau(t)$ and $\lambda(t)=l(t)+i \theta(t)$ the trace and the complex length function respectively of $F(t)$. Since $A \in \mathrm{SO}(2,1), \lambda(A)=\lambda(0) \in(3,+\infty)$ and we denote $\lambda(0)$ by $l_{\alpha}$. We shall need the following Lemma.

Lemma 4.1 Suppose that $F(t)$ is differentiable at 0 . Then

$$
\begin{aligned}
2 \sinh \left(l_{\alpha}\right) \frac{d l}{d t}(0) & =\Re\left(\frac{d \tau}{d t}(0)\right), \\
2\left(\cosh \left(l_{\alpha}\right)-1\right) \frac{d \theta}{d t}(0) & =\Im\left(\frac{d \tau}{d t}(0)\right) .
\end{aligned}
$$


If $F(t)$ is twice differentiable at 0 , then

$$
\begin{aligned}
2 \sinh \left(l_{\alpha}\right) \frac{d^{2} l}{d t^{2}}(0)= & \Re\left(\frac{d^{2} \tau}{d t^{2}}(0)\right)-2 \cosh \left(l_{\alpha}\right)\left(\frac{d l}{d t}(0)\right)^{2} \\
& +2\left(\cosh \left(l_{\alpha}\right)+2\right)\left(\frac{d \theta}{d t}(0)\right)^{2}, \\
4 \sinh ^{2}\left(l_{\alpha} / 2\right) \frac{d^{2} \theta}{d t^{2}}(0)= & \Im\left(\frac{d^{2} \tau}{d t^{2}}(0)\right)-4 \sinh \left(l_{\alpha}\right)\left(\frac{d l}{d t}(0)\right)\left(\frac{d \theta}{d t}(0)\right) .
\end{aligned}
$$

Proof By differentiating

$$
\tau=e^{\lambda}+e^{\bar{\lambda}-\lambda}+e^{-\bar{\lambda}}
$$

with respect to $t$ we obtain

$$
\frac{d \tau}{d t}=c(\lambda) \frac{d \lambda}{d t}-c(-\bar{\lambda}) \frac{d \bar{\lambda}}{d t}
$$

where $c(\lambda)=e^{\lambda}-e^{\bar{\lambda}-\lambda}$. Now

$$
\frac{d \tau}{d t}(0)=c\left(l_{\alpha}\right) \frac{d \lambda}{d t}(0)-c\left(-l_{\alpha}\right) \frac{d \bar{\lambda}}{d t}(0)
$$

The first two equations of the Lemma follow after taking real and imaginary parts in both sides of (4-2).

We next differentiate (4-1) with respect to $t$. We have

$$
\begin{aligned}
\frac{d^{2} \tau}{d t^{2}}= & c(\lambda) \frac{d^{2} \lambda}{d t^{2}}-c(-\bar{\lambda}) \frac{d^{2} \bar{\lambda}}{d t^{2}}+\frac{d c(\lambda)}{d t} \frac{d \lambda}{d t}-\frac{d c(-\bar{\lambda})}{d t} \frac{d \bar{\lambda}}{d t} \\
= & c(\lambda) \frac{d^{2} \lambda}{d t^{2}}-c(-\bar{\lambda}) \frac{d^{2} \bar{\lambda}}{d t^{2}} \\
& +\left(e^{\lambda}+e^{\bar{\lambda}-\lambda}\right)\left(\frac{d \lambda}{d t}\right)^{2}+\left(e^{-\bar{\lambda}}+e^{\bar{\lambda}-\lambda}\right)\left(\frac{d \bar{\lambda}}{d t}\right)^{2}-2 e^{\bar{\lambda}-\lambda}\left|\frac{d \lambda}{d t}\right|^{2},
\end{aligned}
$$

from which we obtain

$$
\begin{aligned}
\frac{d^{2} \tau}{d t^{2}}(0)= & c\left(l_{\alpha}\right) \frac{d^{2} \lambda}{d t^{2}}(0)-c\left(-l_{\alpha}\right) \frac{d^{2} \bar{\lambda}}{d t^{2}}(0) \\
& +\left(e^{l_{\alpha}}+1\right)\left(\frac{d \lambda}{d t}(0)\right)^{2}+\left(e^{-l_{\alpha}}+1\right)\left(\frac{d \bar{\lambda}}{d t}(0)\right)^{2}-2\left|\frac{d \lambda}{d t}(0)\right|^{2}
\end{aligned}
$$

Again, by taking real and imaginary parts in both sides of the above equation, the latter two formulae of the Lemma follow. 
In what follows we shall define a particular deformation suitable for our purposes and give formulae for the derivatives of its complex hyperbolic length. To do this, we consider three loxodromic elements $A, C_{1}, C_{2}$ of $\mathrm{SO}(2,1)$ for which we shall assume that the real axes $\alpha=(r, a)$ of $A$ and the real axes $\gamma_{k}=\left(r_{k}, a_{k}\right)$ of $C_{k}$ have geodesic lengths $2 l_{\alpha}$ and $2 l_{k}, k=1,2$ respectively and that $\alpha$ intersects $\gamma_{k}$ in oriented angles $\phi_{k}=\phi\left(\alpha, \gamma_{k}\right), k=1,2$. Since $A, C_{1}, C_{2} \in \mathrm{SO}(2,1)$, all these axes lie in the standard real Lagrangian plane $R_{\mathbb{R}}$. Let also $2 d$ be the geodesic distance of $\gamma_{1}$ and $\gamma_{2}$ along $\alpha$. We may normalise so that

$$
\mathbf{r}=\left[\begin{array}{l}
0 \\
0 \\
1
\end{array}\right], \quad \mathbf{a}=\left[\begin{array}{l}
1 \\
0 \\
0
\end{array}\right]
$$

with polar vector $\mathbf{n}=\left[\begin{array}{lll}0 & 1 & 0\end{array}\right]^{T}$ and

$$
\mathbf{r}_{k}=\frac{1}{2}\left[\begin{array}{c}
-\exp \left(-d_{k 2}\right)\left(1-\cos \left(\phi_{k}\right)\right) \\
\sqrt{2} \sin \left(\phi_{i}\right) \\
\exp \left(d_{k 2}\right)\left(1+\cos \left(\phi_{k}\right)\right)
\end{array}\right], \quad \mathbf{a}_{k}=\frac{1}{2}\left[\begin{array}{c}
\exp \left(-d_{k 2}\right)\left(1+\cos \left(\phi_{k}\right)\right) \\
\sqrt{2} \sin \left(\phi_{k}\right) \\
-\exp \left(d_{k 2}\right)\left(1-\cos \left(\phi_{k}\right)\right)
\end{array}\right]
$$

with polar vectors

$$
\mathbf{n}_{k}=\left[-\frac{\sqrt{2} \exp \left(-d_{k 2}\right)}{2} \sin \left(\phi_{k}\right) \cos \left(\phi_{k}\right)-\frac{\sqrt{2} \exp \left(d_{k 2}\right)}{2} \sin \left(\phi_{k}\right)\right]^{T}, \quad k=1,2
$$

respectively. Here we have set and $d_{12}=d, d_{22}=0$. Hence, it follows from the discussion in Section 2.4 that we may write $A=E\left(l_{\alpha}\right)$ and for $k=1,2$

$$
C_{k}=Q_{k} E\left(l_{k}\right) Q_{k}^{-1}, \quad Q_{k}=\left[\begin{array}{lll}
\mathbf{a}_{k} & \mathbf{n}_{k} & \mathbf{r}_{k}
\end{array}\right] .
$$

Let $\zeta_{k} \in \mathbb{C}$, and $\varepsilon>0$ such that for each $t \in \Delta_{\varepsilon}$ we have $t \zeta_{k} \in S \cup(-S), k=1,2$. Here $S$ is the strip as in (2-1) and $-S$ is the domain induced by reflection of $S$ on the imaginary axis. For $t \in \Delta_{\varepsilon}$ consider $B_{k}=Q_{k} E\left(t \zeta_{k}\right) Q_{k}^{-1}, k=1,2$. If $t \zeta_{k} \in S$ then $B_{k}$ is a loxodromic element of $\mathrm{SU}(2,1)$ with complex hyperbolic length $t \zeta_{k}$ and real axis $\gamma_{k}$. If $t \zeta_{k} \in-S$ then $B_{k}$ is a loxodromic element of $\mathrm{SU}(2,1)$ with complex hyperbolic length $-t \zeta_{k}$ and real axis $-\gamma_{k}$, that is the real axis of $C_{k}$ with the opposite orientation.

We now define

$$
G(t)=\prod_{k=1}^{2} Q_{k} E\left(t \zeta_{k}\right) Q_{k}^{-1} \cdot E\left(l_{\alpha}\right)
$$

It is clear that $G$ is a deformation of $A$ into $\mathrm{SU}(2,1)$. Denote as before by $\tau(t)$ the trace and by $\lambda(t)=l(t)+i \theta(t)$ the complex length of $G(t)$ respectively. 
Theorem 4.2 (First derivatives.) The complex hyperbolic length function $\lambda(t)=$ $l(t)+i \theta(t)$ of the deformation $G(t)$ given in (4-3) is a real differentiable function at 0 . The first derivatives of $l$ and $\theta$ at 0 are given by

$$
\begin{aligned}
& \frac{d l}{d t}(0)=\sum_{k=1}^{2} \Re\left(\zeta_{k}\right) \cdot \cos \left(\phi_{k}\right), \\
& \frac{d \theta}{d t}(0)=\sum_{k=1}^{2} \Im\left(\zeta_{k}\right) \cdot \frac{3 \cos ^{2}\left(\phi_{k}\right)-1}{2} .
\end{aligned}
$$

Proof We differentiate (4-3) with respect to $t$ at 0 to obtain

$$
\frac{d G}{d t}(0)=\sum_{k=1}^{2} Q_{k} D\left(\zeta_{k}\right) Q_{k}^{-1} E\left(l_{\alpha}\right)
$$

Here

$$
\begin{aligned}
D\left(\zeta_{k}\right)=\frac{d E(t \zeta)}{d t}(0) & =\left[\begin{array}{ccc}
\zeta_{k} & 0 & 0 \\
0 & \zeta_{k}-\zeta_{k} & 0 \\
0 & 0 & -\overline{\zeta_{k}}
\end{array}\right] \\
& =\Re\left(\zeta_{k}\right) \cdot\left[\begin{array}{ccc}
1 & 0 & 0 \\
0 & 0 & 0 \\
0 & 0 & -1
\end{array}\right]+i \Im\left(\zeta_{k}\right) \cdot\left[\begin{array}{ccc}
1 & 0 & 0 \\
0 & -2 & 0 \\
0 & 0 & 1
\end{array}\right] \\
& =\Re\left(\zeta_{k}\right) \cdot D_{1}+i \Im\left(\zeta_{k}\right) \cdot D_{2}, \quad k=1,2 .
\end{aligned}
$$

Now,

$$
\begin{aligned}
& \Re\left(\frac{d \tau}{d t}(0)\right)=\Re\left(\frac{d \operatorname{tr}(G)}{d t}(0)\right)=\Re\left(\operatorname{tr}\left(\frac{d G}{d t}(0)\right)\right) \\
& =\sum_{k=1}^{2} \Re\left(\zeta_{k}\right) \cdot \operatorname{tr}\left(Q_{k} D_{1} Q_{k}^{-1} E\left(l_{\alpha}\right)\right) \\
& =\sum_{k=1}^{2} \Re\left(\zeta_{k}\right) \cdot \operatorname{tr}\left(\left[\begin{array}{ccc}
\cos \left(\phi_{k}\right) e^{l_{\alpha}} & * & * \\
* & 0 & * \\
* & * & -\cos \left(\phi_{k}\right) e^{-l_{\alpha}}
\end{array}\right]\right) \\
& =2 \sinh \left(l_{\alpha}\right) \sum_{k=1}^{2} \Re\left(\zeta_{k}\right) \cdot \cos \left(\phi_{k}\right) .
\end{aligned}
$$


Similarly,

$$
\begin{aligned}
& \Im\left(\frac{d \tau}{d t}(0)\right)=\Im\left(\frac{d \operatorname{tr}(G)}{d t}(0)\right) \\
& =\sum_{k=1}^{2} \Im\left(\zeta_{k}\right) \cdot \operatorname{tr}\left(Q_{k} D_{2} Q_{k}^{-1} E\left(l_{\alpha}\right)\right) \\
& =\sum_{k=1}^{2} \Im\left(\zeta_{k}\right) \cdot \operatorname{tr}\left(\left[\begin{array}{ccc}
\frac{3 \cos ^{2}\left(\phi_{k}\right)-1}{2} \cdot e^{l_{\alpha}} & * & * \\
* & 3 \cos ^{2}\left(\phi_{k}\right)-1 & * \\
* & * & \frac{3 \cos ^{2}\left(\phi_{k}\right)-1}{2} \cdot e^{-l_{\alpha}}
\end{array}\right]\right) \\
& =\left(\cosh \left(l_{\alpha}\right)-1\right) \sum_{k=1}^{2} \Im\left(\zeta_{k}\right) \cdot\left(3 \cos ^{2}\left(\phi_{k}\right)-1\right) .
\end{aligned}
$$

The desired formulae now follow directly from Lemma 4.1.

Theorem 4.3 (Second derivatives.) The complex hyperbolic length function $\lambda(t)=$ $l(t)+i \theta(t)$ of the deformation $G(t)$ is a twice real differentiable function at 0 . Moreover, the following hold.

$$
\begin{aligned}
& \frac{d^{2} l}{d t^{2}}(0)=\frac{1}{2 \sinh \left(l_{\alpha} / 2\right)} \sum_{k, l=1}^{2} \Re\left(\zeta_{k}\right) \Re\left(\zeta_{l}\right) \cdot \cosh \left(\frac{l_{\alpha}}{2}-d_{k l}\right) \sin \left(\phi_{k}\right) \sin \left(\phi_{l}\right) \\
& -\frac{9}{2 \sinh \left(l_{\alpha} / 2\right)} \sum_{k, l=1}^{2} \Im\left(\zeta_{k}\right) \Im\left(\zeta_{l}\right) \cdot \cos \left(\frac{l_{\alpha}}{2}-d_{k l}\right) \cos \left(\phi_{k}\right) \cos \left(\phi_{l}\right) \sin \left(\phi_{k}\right) \sin \left(\phi_{l}\right) \\
& -\frac{9}{4 \sinh \left(l_{\alpha}\right)} \sum_{k, l=1}^{2} \Im\left(\zeta_{k}\right) \Im\left(\zeta_{l}\right) \cdot \cosh \left(l_{\alpha}-2 d_{k l}\right) \sin ^{2}\left(\phi_{k}\right) \sin ^{2}\left(\phi_{l}\right), \\
& \frac{d^{2} \theta}{d t^{2}}(0) 3 \frac{\cosh ^{2}\left(l_{\alpha} / 2\right)}{\sinh ^{2}\left(l_{\alpha} / 2\right)} \sum_{k, l=1}^{2} \epsilon_{k l} \Re\left(\zeta_{k}\right) \Im\left(\zeta_{l}\right) \cdot \sinh \left(\frac{l_{\alpha}}{2}-d_{k l}\right) \cos \left(\phi_{k}\right) \sin \left(\phi_{k}\right) \sin \left(\phi_{l}\right) .
\end{aligned}
$$

Here,

$$
\epsilon_{k l}=\left\{\begin{array}{cc}
1 & k=l \\
-1 & k \neq l
\end{array}, \quad d_{k l}=\left\{\begin{array}{ll}
0 & k=l \\
d & k \neq l
\end{array}\right. \text {. }\right.
$$


Proof From Theorem 4.2 we have

$$
\begin{aligned}
& \frac{d l}{d t}(0)=\sum_{k=1}^{2} \Re\left(\zeta_{k}\right) \cdot \cos \left(\phi_{k}\right), \\
& \frac{d \theta}{d t}(0)=\sum_{k=1}^{2} \Im\left(\zeta_{k}\right) \cdot \frac{3 \cos ^{2}\left(\phi_{k}\right)-1}{2} .
\end{aligned}
$$

We now differentiate (4-3) twice with respect to $t$. At 0 we have

$$
\frac{d^{2} G}{d t^{2}}(0)=\sum_{k=1}^{2} Q_{k} D^{2}\left(\zeta_{k}\right) Q_{k}^{-1} \cdot E\left(l_{\alpha}\right)+2 \prod_{k=1}^{2} Q_{k} D\left(\zeta_{k}\right) Q_{k}^{-1} \cdot E\left(l_{\alpha}\right) .
$$

We set for convenience

$$
R_{1}=\sum_{k=1}^{2} Q_{k} D^{2}\left(\zeta_{k}\right) Q_{k}^{-1} \cdot E\left(l_{\alpha}\right), \quad R_{2}=\prod_{k=1}^{2} Q_{k} D\left(\zeta_{k}\right) Q_{k}^{-1} \cdot E\left(l_{\alpha}\right) .
$$

Consider the matrices $D_{1}$ and $D_{2}$ as in the proof of Theorem 4.2. It is easy to see that for $k=1,2$ the following hold.

$$
D^{2}\left(\zeta_{k}\right)=\Re^{2}\left(\zeta_{k}\right) \cdot D_{1}^{2}-\Im^{2}\left(\zeta_{k}\right) \cdot D_{2}^{2}+2 i \Re\left(\zeta_{k}\right) \Im\left(\zeta_{k}\right) \cdot D_{1} D_{2}
$$

where

$$
D_{1}^{2}=\left[\begin{array}{lll}
1 & 0 & 0 \\
0 & 0 & 0 \\
0 & 0 & 1
\end{array}\right], \quad D_{2}^{2}=\left[\begin{array}{lll}
1 & 0 & 0 \\
0 & 4 & 0 \\
0 & 0 & 1
\end{array}\right], \quad D_{1} D_{2}=\left[\begin{array}{ccc}
1 & 0 & 0 \\
0 & 0 & 0 \\
0 & 0 & -1
\end{array}\right]=D_{1}
$$

Thus we write

$$
\begin{aligned}
\operatorname{tr}\left(R_{1}\right)= & \sum_{k=1}^{2} \Re^{2}\left(\zeta_{k}\right) \cdot \operatorname{tr}\left(Q_{k} D_{1}^{2} Q_{k}^{-1} E\left(l_{\alpha}\right)\right)-\Im^{2}\left(\zeta_{k}\right) \cdot \operatorname{tr}\left(Q_{k} D_{2}^{2} Q_{k}^{-1} E\left(l_{\alpha}\right)\right) \\
& -2 i \sum_{k=1}^{2} \Re\left(\zeta_{k}\right) \Im\left(\zeta_{k}\right) \cdot \operatorname{tr}\left(Q_{k} D_{1} Q_{k}^{-1} E\left(l_{\alpha}\right)\right),
\end{aligned}
$$

and simple calculations yield the following.

$$
\begin{aligned}
\Re\left(\operatorname{tr}\left(R_{1}\right)\right) & =\sum_{k=1}^{2} \Re^{2}\left(\zeta_{k}\right) \cdot\left(\left(\left(1+\cos ^{2}\left(\phi_{k}\right)\right) \cosh \left(l_{\alpha}\right)+\sin ^{2}\left(\phi_{k}\right)\right)\right. \\
& -\sum_{k=1}^{2} \Im^{2}\left(\zeta_{k}\right) \cdot\left(\left(5-3 \cos ^{2}\left(\phi_{k}\right)\right) \cosh \left(l_{0}\right)+1-3 \cos ^{2}\left(\phi_{k}\right)\right),
\end{aligned}
$$




$$
\Im\left(\operatorname{tr}\left(R_{1}\right)\right)=4 \sum_{k=1}^{2} \Re\left(\zeta_{k}\right) \Im\left(\zeta_{k}\right) \cdot \cos \left(\phi_{k}\right) \sinh \left(l_{\alpha}\right) .
$$

On the other hand we have

$$
\begin{aligned}
\operatorname{tr}\left(R_{2}\right)= & \Re\left(\zeta_{1}\right) \Re\left(\zeta_{2}\right) \cdot \operatorname{tr}\left(Q_{1} D_{1} Q_{1}^{-1} Q_{2} D_{1} Q_{2}^{-1} E\left(l_{\alpha}\right)\right) \\
& -\Im\left(\zeta_{1}\right) \Im\left(\zeta_{2}\right) \cdot \operatorname{tr}\left(Q_{1} D_{2} Q_{1}^{-1} Q_{2} D_{2} Q_{2}^{-1} E\left(l_{\alpha}\right)\right) \\
& +i \Re\left(\zeta_{1}\right) \Im\left(\zeta_{2}\right) \cdot \operatorname{tr}\left(Q_{1} D_{1} Q_{1}^{-1} Q_{2} D_{2} Q_{2}^{-1} E\left(l_{\alpha}\right)\right) \\
& +i \Im\left(\zeta_{1}\right) \Re\left(\zeta_{2}\right) \cdot \operatorname{tr}\left(Q_{1} D_{2} Q_{1}^{-1} Q_{2} D_{1} Q_{2}^{-1} E\left(l_{\alpha}\right)\right)
\end{aligned}
$$

We set

$$
\begin{array}{ll}
R_{2}^{1}=Q_{1} D_{1} Q_{1}^{-1} Q_{2} D_{1} Q_{2}^{-1} E\left(l_{\alpha}\right), & R_{2}^{2}=Q_{1} D_{2} Q_{1}^{-1} Q_{2} D_{2} Q_{2}^{-1} E\left(l_{\alpha}\right) \\
T_{2}^{1}=Q_{1} D_{1} Q_{1}^{-1} Q_{2} D_{2} Q_{2}^{-1} E\left(l_{\alpha}\right), & T_{2}^{2}=Q_{1} D_{2} Q_{1}^{-1} Q_{2} D_{1} Q_{2}^{-1} E\left(l_{\alpha}\right) .
\end{array}
$$

Straightforward calculations then yield

$$
\begin{aligned}
\operatorname{tr}\left(R_{2}^{1}\right)= & 2 \cos \left(\phi_{1}\right) \cos \left(\phi_{2}\right) \cosh \left(l_{\alpha}\right) \\
& +2 \cosh \left(l_{\alpha} / 2\right) \cosh \left(\frac{l_{\alpha}}{2}-d_{12}\right) \sin \left(\phi_{1}\right) \sin \left(\phi_{2}\right) \\
\operatorname{tr}\left(R_{2}^{2}\right)= & 2\left(3 \cos ^{2}\left(\phi_{1}\right)-1\right)\left(3 \cos ^{2}\left(\phi_{2}\right)-1\right)\left(2-\cosh \left(l_{\alpha}\right)\right) \\
& +18 \cosh \left(l_{\alpha} / 2\right) \cosh \left(\frac{l_{\alpha}}{2}-d_{12}\right) \sin \left(\phi_{1}\right) \sin \left(\phi_{2}\right) \cos \left(\phi_{1}\right) \cos \left(\phi_{2}\right) \\
& +\frac{9}{2} \cosh \left(l_{\alpha}-2 d_{12}\right) \sin ^{2}\left(\phi_{1}\right) \sin ^{2}\left(\phi_{2}\right) \\
\operatorname{tr}\left(T_{2}^{1}\right)= & \sinh \left(l_{\alpha}\right) \cos \left(\phi_{1}\right)\left(3 \cos ^{2}\left(\phi_{2}\right)-1\right) \\
& -6 \sinh \left(\frac{l_{\alpha}}{2}-d_{12}\right) \cosh \left(l_{\alpha} / 2\right) \sin \left(\phi_{1}\right) \sin \left(\phi_{2}\right) \cos \left(\phi_{2}\right) \\
\operatorname{tr}\left(T_{2}^{2}\right)= & \sinh \left(l_{\alpha}\right) \cos \left(\phi_{2}\right)\left(3 \cos ^{2}\left(\phi_{1}\right)-1\right) \\
& -6 \sinh \left(\frac{l_{\alpha}}{2}-d_{12}\right) \cosh \left(l_{\alpha} / 2\right) \sin \left(\phi_{1}\right) \sin \left(\phi_{2}\right) \cos \left(\phi_{1}\right)
\end{aligned}
$$

Thus from the relations

$$
\begin{aligned}
& \Re\left(\frac{d^{2} \tau}{d t^{2}}(0)\right)=\Re\left(\operatorname{tr}\left(R_{1}\right)\right)+2 \Re\left(\operatorname{tr}\left(R_{2}\right)\right), \\
& \Im\left(\frac{d^{2} \tau}{d t^{2}}(0)\right)=\Im\left(\operatorname{tr}\left(R_{1}\right)\right)+2 \Im\left(\operatorname{tr}\left(R_{2}\right)\right)
\end{aligned}
$$


and after summing up, by making use of Lemma 4.1 the desired equations follow. The proof is thus complete.

\subsection{Complex hyperbolic Wolpert-Kerckhoff formulae}

Let $\rho_{0}$ be our fixed $\mathbb{R}$-Fuchsian point, and let $A=\rho_{0}(a) \in \Gamma_{0}$ be such that its real axis is $\alpha$ and its geodesic length is $2 l_{\alpha}$. Let also $\rho_{t}, t \in \Delta_{\varepsilon}$ be the complex hyperbolic quakebend homomorphism associated to $\rho_{0}$ as in Theorem 3.9, namely

$$
\rho_{t}(a)=B_{t}\left(O, \rho_{0}(a)(O)\right) \rho_{0}(a), \quad t \in \Delta_{\varepsilon} .
$$

By Proposition $3.8 B_{t}$ varies real analytically with $t$. The trace function as well as the complex hyperbolic length function of $\rho_{t}(a)$ shall be denoted as usual by $\tau(t)=\operatorname{tr}\left(\rho_{t}(a)\right)$ and $\lambda(t)=\lambda\left(\rho_{t}(a)\right)$ respectively. Set $\lambda(t)=l(t)+i \theta(t)$. We have $\lambda(0)=l(0)=l_{\alpha}$.

The corresponding deformation of $A$ is

$$
B(t)=\prod_{k=1}^{n} Q_{k} E\left(t \zeta_{k}\right) Q_{k}^{-1} \cdot A .
$$

Taking the derivatives at 0 we have

$$
\begin{aligned}
\frac{d B}{d t}(0) & =\sum_{k=0}^{n} Q_{k}\left(\frac{d}{d t} E\left(t \zeta_{k}\right)\right)(0) Q_{k}^{-1} A \\
\frac{d^{2} B}{d t^{2}}(0) & =\sum_{k=1}^{n} Q_{k}\left(\frac{d^{2}}{d t^{2}} E\left(t \zeta_{k}\right)\right)(0) Q_{k}^{-1} A \\
& +\sum_{k, l=0}^{n} Q_{k}\left(\frac{d}{d t} E\left(t \zeta_{k}\right)\right)(0) Q_{k}^{-1} Q_{l}\left(\frac{d}{d t} E\left(t \zeta_{l}\right)\right)(0) Q_{l}^{-1} A
\end{aligned}
$$

Theorem 4.2 and Theorem 4.3 apply in this case and we immediately obtain the following Theorem.

Theorem 4.4 Let $\rho_{0} \in Q_{\mathbb{C}}$ be an $\mathbb{R}-$ Fuchsian point and let $(\Lambda, \mu)$ be a finite geodesic lamination with transverse measure in $\Sigma_{0}=\mathbf{H}_{\mathbb{C}}^{2} / \Gamma_{0}, \Gamma_{0}=\rho_{0}\left(\pi_{1}\right)$. Let $\alpha \in \Sigma_{0}$ be a geodesic intersecting the leaves $\gamma_{1}, \ldots, \gamma_{n}$ of $\Lambda$ in oriented angles $\phi_{k}, k=1, \ldots, n$. Then the complex hyperbolic length function $\lambda(t)=l(t)+i \theta(t)$ is differentiable at 0 
and moreover,

$$
\begin{aligned}
& \frac{d l}{d t}(0)=\sum_{k=1}^{n} \Re\left(\zeta_{k}\right) \cdot \cos \left(\phi_{k}\right), \\
& \frac{d \theta}{d t}(0)=\sum_{k=1}^{n} \Im\left(\zeta_{k}\right) \cdot \frac{3 \cos ^{2}\left(\phi_{k}\right)-1}{2} .
\end{aligned}
$$

Theorem 4.5 With the assumptions of Theorem 4.4, the complex hyperbolic length function $\lambda(t)=l(t)+i \theta(t)$ is twice differentiable at 0 and moreover,

$$
\begin{aligned}
& \frac{d^{2} l}{d t^{2}}(0)=\frac{1}{2 \sinh \left(l_{\alpha} / 2\right)} \sum_{k, l=1}^{n} \Re\left(\zeta_{k}\right) \Re\left(\zeta_{l}\right) \cdot \cosh \left(\frac{l_{\alpha}}{2}-d_{k l}\right) \sin \left(\phi_{k}\right) \sin \left(\phi_{l}\right) \\
& -\frac{9}{2 \sinh \left(l_{\alpha} / 2\right)} \sum_{k, l=1}^{n} \Im\left(\zeta_{k}\right) \Im\left(\zeta_{l}\right) \cdot \cos \left(\frac{l_{\alpha}}{2}-d_{k l}\right) \cos \left(\phi_{k}\right) \cos \left(\phi_{l}\right) \sin \left(\phi_{k}\right) \sin \left(\phi_{l}\right) \\
& -\frac{9}{4 \sinh \left(l_{\alpha}\right)} \sum_{k, l=1}^{n} \Im\left(\zeta_{k}\right) \Im\left(\zeta_{l}\right) \cdot \cosh \left(l_{\alpha}-2 d_{k l}\right) \sin ^{2}\left(\phi_{k}\right) \sin ^{2}\left(\phi_{l}\right), \\
& \frac{d^{2} \theta}{d t^{2}}(0)=3 \frac{\cosh \left(l_{\alpha} / 2\right)}{\sinh ^{2}\left(l_{\alpha} / 2\right)} \sum_{k, l=1}^{n} \epsilon_{k l} \Re\left(\zeta_{k}\right) \Im\left(\zeta_{l}\right) \cdot \sinh \left(\frac{l_{\alpha}}{2}-d_{k l}\right) \cos \left(\phi_{k}\right) \sin \left(\phi_{k}\right) \sin \left(\phi_{l}\right) .
\end{aligned}
$$

Here,

$$
\epsilon_{k l}=\left\{\begin{array}{cc}
1 & k=l \\
-1 & k \neq l
\end{array}\right.
$$

and $2 d_{k l}$ is the distance along $\alpha$ from $\gamma_{k} \cap \alpha$ to $\gamma_{l} \cap \alpha$. $\left(d_{k k}=0\right)$.

We wish to further comment these results. Formula (4-6) is a generalisation of Kerckhoff's formula; see [6]. Formula (4-7) has no analogue in the classical case. The following cases are also of special interest.

A. $\mu$ is real, corresponding to pure earthquake.

Then $\theta=0$ identically on the bending curve, since the deformation is in $\operatorname{SO}(2,1)$. On the other hand, the first derivative of $l$ at zero is

(1) positive in the case of left earthquakes $\left(\Re\left(\zeta_{k}\right)>0\right)$, or

(2) negative in the case of right earthquakes $\left(\Re\left(\zeta_{k}\right)<0\right)$.

The second derivative of $l$ is positive for both pure left and pure right earthquakes. This is entirely consistent with the classical case and implies likewise that $l$ is convex on earthquake paths, see [6]. 
B. $\mu$ is purely imaginary, corresponding to pure bending.

Then as in the classical case, the first derivative of $l$ is zero and the second derivative of $l$ is negative. Therefore $l$ attains a local maximum at $t=0$.

The derivative of $\theta$ at zero is

(1) positive if the bending angles are positive and $3 \cos ^{2}\left(\phi_{k}\right)>1$ or the bending angles are negative and $3 \cos ^{2}\left(\phi_{k}\right)<1$, and

(2) negative if the bending angles are positive and $3 \cos ^{2}\left(\phi_{k}\right)<1$ or the bending angles are negative and $3 \cos ^{2}\left(\phi_{k}\right)>1$.

\section{References}

[1] B Apanasov, Bending deformations of complex hyperbolic surfaces, J. Reine Angew. Math. 492 (1997) 75-91 MR1488065

[2] D B A Epstein, A Marden, Convex hulls in hyperbolic space, a theorem of Sullivan, and measured pleated surfaces, from: "Analytical and geometric aspects of hyperbolic space (Coventry/Durham, 1984)", London Math. Soc. Lecture Note Ser. 111, Cambridge Univ. Press, Cambridge (1987) 113-253 MR903852

[3] E Falbel, R A Wentworth, Eigenvalues of products of unitary matrices and Lagrangian involutions, Topology 45 (2006) 65-99 MR2170495

[4] W M Goldman, Complex hyperbolic geometry, Oxford Mathematical Monographs, The Clarendon Press Oxford University Press (1999) MR1695450

[5] W M Goldman, M Kapovich, B Leeb, Complex hyperbolic manifolds homotopy equivalent to a Riemann surface, Comm. Anal. Geom. 9 (2001) 61-95 MR1807952

[6] S P Kerckhoff, The Nielsen realization problem, Ann. of Math. (2) 117 (1983) 235-265 MR690845

[7] C Kourouniotis, Deformations of hyperbolic structures, Math. Proc. Cambridge Philos. Soc. 98 (1985) 247-261 MR795891

[8] C Kourouniotis, Bending in the space of quasi-Fuchsian structures, Glasgow Math. J. 33 (1991) 41-49 MR1089952

[9] C Kourouniotis, The geometry of bending quasi-Fuchsian groups, from: "Discrete groups and geometry (Birmingham, 1991)", London Math. Soc. Lecture Note Ser. 173, Cambridge Univ. Press, Cambridge (1992) 148-164 MR1196924

[10] J R Parker, ID Platis, Open sets of maximal dimension in complex hyperbolic quasiFuchsian space, J. Differential Geom. 73 (2006) 319-350 MR2226956

[11] J R Parker, ID Platis, Complex hyperbolic Fenchel-Nielsen coordinates, Topology (2007) To appear. 
[12] J R Parker, C Series, Bending formulae for convex hull boundaries, J. Anal. Math. 67 (1995) 165-198 MR1383492

[13] I D Platis, Complex symplectic geometry of quasi-Fuchsian space, Geom. Dedicata 87 (2001) 17-34 MR1866841

[14] C Series, An extension of Wolpert's derivative formula, Pacific J. Math. 197 (2001) 223-239 MR1810217

[15] W P Thurston, Three-dimensional geometry and topology. Vol. 1, Princeton Mathematical Series 35, Princeton University Press, Princeton, NJ (1997) MR1435975 Edited by Silvio Levy

[16] D Toledo, Representations of surface groups in complex hyperbolic space, J. Differential Geom. 29 (1989) 125-133 MR978081

[17] P Will, Lagrangian decomposability of some two-generator subgroups of $\mathrm{PU}(2,1), \mathrm{C}$. R. Math. Acad. Sci. Paris 340 (2005) 353-358 MR2127109

[18] S Wolpert, The Fenchel-Nielsen deformation, Ann. of Math. (2) 115 (1982) 501-528 MR657237

[19] S Wolpert, On the symplectic geometry of deformations of a hyperbolic surface, Ann. of Math. (2) 117 (1983) 207-234 MR690844

Department of Mathematics, Aristotle University of Salonica, Salonica, Greece

jplatis@math.uoc.gr

http://www.math.uoc.gr/ jplatis/

Proposed: Jean-Pierre Otal

Received: 23 February 2007

Seconded: Walter Neumann, Benson Farb

Accepted: 6 December 2007 